\title{
COEXistence OF LONG-TERM AND SHORT-TERM CONTRACTS*
}

\author{
Inés Macho-Stadler ${ }^{\dagger} \quad$ David Pérez-Castrillo ${ }^{\ddagger} \quad$ Nicolás Porteiro $^{\S}$ \\ February 7, 2014
}

\begin{abstract}
We study the length of agreements in a market in which infinitely-lived firms contract with agents that live for two periods. Firms differ in the expected values of their projects, as do workers in their abilities to manage projects. Worker effort is not contractible and worker ability is revealed during the relationship. The market dictates the trade-off between sorting and incentives. Short- and long-term contracts often coexist: The best firms always use short-term contracts to hire highability senior workers, firms with less profitable projects use short-term contracts to save on the cost of hiring junior workers, whereas intermediate firms use long-term agreements to provide better incentives to their workers. We relate our results to the optimal assignment literature that follows Becker (1973).
\end{abstract}

${ }^{*}$ We are grateful to the participants at seminars at CREST (Paris), U de Salamanca and U Autònoma de Barcelona and at SAE 2011 (Málaga), 20iDEA 2011 (Barcelona), Games 2012 (Istanbul), CICGTA 2012 (Qingdao), SAET 2013 (Paris), as well as four reviewers and the co-editor for their insightful comments. Financial support from Ministerio de Ciencia y Tecnología (ECO2008-04321, ECO2009-07616 and ECO2012-31962), Generalitat de Catalunya (2009SGR-169), Junta de Andalucía (SEJ-02936 and SEJ04992), Severo Ochoa Programme (SEV2011-0075), and ICREA Academia is gratefully acknowledged.

${ }^{\dagger}$ Corresponding author. Universitat Autonoma de Barcelona and Barcelona GSE; Dept. Economía e Hist. Económica. UAB edifici B; E-08193 Bellaterra (Barcelona, Spain). Email: ines.macho@uab.es.

${ }^{\ddagger}$ Universitat Autonoma de Barcelona and Barcelona GSE; Dept. Economía e Hist. Económica. Email: david.perez@uab.es.

$\S$ Universidad Pablo Olavide; Department of Economics.

ॠNico passed away in April 2012. He was a talented, honest, brave, and enthusiastic friend. We miss him deeply. 
JEL numbers: D86, C78

Keewords: Matching, Moral Hazard, Contracts, Assignment 


\section{Introduction}

A key element of a contract is its duration. Some firms sign a long-term agreement with a worker for several years. Other firms sign contracts period by period, either with the same worker or with different workers over time. In this paper, we analyse the firms' choice of short-term or long-term contracts in a market where heterogeneous firms compete for heterogeneous workers who are subject to moral hazard. ${ }^{1}$

In our dynamic model, firms hire workers to run their projects. Firms are infinitely lived and differ with respect to the profitability of their projects. Workers live for two periods, starting as junior agents in the labour market. In any period, each firm requires two workers: a junior agent (with no specific skills) and a senior, experienced worker, whose expertise is crucial for the advancement of the project. All the participants are risk neutral and have the capacity to commit to long-term contracts. ${ }^{2}$ Workers are protected by limited liability, and a moral hazard problem is present because the contract cannot specify the senior's effort when running a project.

When young, all workers are indistinguishable in ability. Working for a firm as a junior has a training component: workers acquire the knowledge and experience required to run projects as seniors. We assume that this training provides industry-specific human capital, which allows any worker hired by a firm as a junior to run a project as a senior for any firm in the market. Whereas the competence level needed to run a project as a

\footnotetext{
${ }^{1}$ As the contributions by Dam and Pérez-Castrillo (2006), Serfes (2008), and Alonso-Paulí and PérezCastrillo (2012) show, when the analysis is enlarged to include market interactions, the shape of optimal contracts may differ substantially from the agreements obtained for a given relationship studied in isolation. When heterogeneous principals compete for heterogeneous agents, the identity of the partners in each relationship (in addition to the contract signed) is endogenous, as are the levels of utility obtained by the agents.

${ }^{2}$ When it is difficult to ensure commitment from agents, contracts may include, for example, noncompete clauses under which the agent agrees not to pursue a similar profession or trade with a firm in the same industry if he breaks the contract. Contracts may also include other clauses that reduce the worker's mobility by increasing the cost of the worker being hired by another firm. If no participant can commit to a long-term contract, then all contracts must be short-term. If the participants on one side of the market (say, the firms) can commit but the others cannot, then there can still be room for long-term contracts, but they are typically less efficient than in an environment with full commitment. In terms of the commitment possibilities, we place ourselves in the best scenario for the prevalence of long-term contracts.
} 
senior is unknown to all market participants ex-ante, it is revealed during the training period: after the agent has worked for a firm as a junior, all firms and the worker himself discover whether he has high or low ability as a senior. In other words, we model a very simple training technology that combines two dimensions of learning. First, the innate ability of the worker is revealed through his training as a junior worker, with this information becoming common knowledge for the industry. Second, there is a learningby-doing component: working as a junior is a prerequisite for subsequently running a project as a senior.

We characterise the equilibrium of this model. Our equilibrium concept is close to the idea of "stability" used in the matching literature that analyses contracts in environments where matching between firms and workers is endogenous. ${ }^{3}$ To be at equilibrium, an outcome (that is, a matching and a set of contracts) must be immune to deviations by participants.

We first show that firms signing long-term contracts in equilibrium offer a young agent an agreement in every period that includes a low salary for the first period together with the promise of a higher reward when he becomes senior. This practice allows firms to alleviate the incentive problem they face with senior agents, improving the efficiency of the relationship, and their profits. Firms that sign short-term contracts hire a junior agent every period with no promise of a future position. They also sign short-term contracts every period with a senior worker (who may or may not be the same worker they hired as a junior in the previous period); the terms of the agreement will depend on the senior worker's value in the market (which may depend on the worker's ability level).

Second, we show that the market equilibrium depends on the characteristics of the set of firms considered. When all firms in the market have very profitable projects, all of them sign long-term contracts in equilibrium. Each firm offers the same contract that it would offer in an agency problem (no market) situation. ${ }^{4}$

\footnotetext{
${ }^{3}$ Stability and competitive equilibrium are very closely related concepts. Any stable outcome is also a competitive equilibrium and vice-versa. For (early) matching models in which the parties decide on money instead of contracts, see the original contribution by Shapley and Shubik (1972) and the excellent literature review by Roth and Sotomayor (1990).

${ }^{4}$ In an isolated long-term relationship between a firm (principal) and a worker (agent), if both are able to commit to the duration of the contract, the solution to the repeated moral hazard problem is to offer a long-term contract (see, e.g., Lambert, 1983, Rogerson, 1985, Malcomson and Spinnewyn, 1988,
} 
More interestingly, when heterogeneity in the population of firms is sufficiently large, some firms sign short-term contracts with their junior workers and recruit in the market for senior workers. These firms specialise in a particular type of senior: firms with the most profitable projects hire high-ability senior agents, whereas those with the least profitable ventures employ low-ability senior workers.

The firms with highly profitable projects obtain large profits by hiring high-ability senior agents and are willing to offer high wages in the market to attract them to run their projects. As a result, the expected utility of junior workers, when they accept shortterm contracts, is high because, if they turn out to be of high ability, they will obtain a reward in the market as seniors. This prospective reward leads workers to accept low wages as juniors. Firms with relatively poor projects take advantage of this willingness of junior workers to accept low wages during the training period. For these firms, the savings they achieve on juniors' wages is more important than the losses they incur from hiring low-ability seniors. Therefore, in these equilibria, the firms with the most profitable projects use short-term contracts to obtain the services of high-ability workers, whereas the firms with the least profitable projects use short-term contracts to economise on the costs of junior workers. In this sense, the matching between firms and senior agents is positive assortative for the set of firms that use short-term contracts. Finally, for firms with intermediate projects, the trade-off between the advantages of long- and short-term contracts is often resolved in favour of long-term contracts.

The results and intuitions of our model can be applied to industries where talent (agents' ability) can be publicly assessed and is not firm-specific. These industries are also analysed by Terviö (2009), who finds that under short-term contracts, firms rely too much on the pool of incumbent workers, that there is market failure in the discovery of talent, and that wages for known talents are too high. Our focus is not on the discovery of talent but on the market assignment of workers to firms, in particular through the choice of contract length. ${ }^{5}$

The arts and sports are examples of markets in which the abilities of senior workers and Chiappori et al., 1994).

${ }^{5}$ As Terviö (2009) observes, in his model, "inexperienced individuals would pay for the chance to discover their talent". In our model, junior workers implicitely pay to discover their talent by accepting a short-term low-wage contract. This reduction in the wage has implications not only for the workers and the firms that are ready to pay for talent but also for other firms that benefit from the low salary. 
are widely known, as they are subject to public scrutiny through the performance of these workers, and the human capital involved is mainly industry specific. In these markets, the length of the agreement between companies or clubs and agents is as important as the level of compensation. Moreover, some long-term contracts include clauses that prevent or make it difficult to break them. Actors, singers or soccer players, for instance, may sign exclusive contracts (with a studio, a record company or a club) for a long period during which they cannot perform, record or play for another firm. ${ }^{6}$ However, there are also companies that offer shorter contracts, particularly to young singers or soccer players. The stars of these markets (relatively few individuals) attain prominence and success, and earnings are significantly greater than those of ordinary workers. The same can be said for surgeons or creatives in the advertising industry. Finally, the market for upperlevel executives, or top scientists and engineers, also shares some similar features: these executives are well-known within their industry, and their contracts may (or may not) include special clauses that function as commitment devices intended to prevent them from moving to other firms.

To the best of our knowledge, ours is the first paper to study how the choice of contract length may be determined by market interactions. The paper is complementary to the contribution of Ghosh and Waldman (2010), who compare two contractual arrangements: up-or-stay vs. up-or-out contracts in a setting where multiple firms compete for one worker. Their paper does not address the issue of endogenous matching, as it studies firms' attempts, through Bertrand competition in wages, to attract the single worker available in the market. They show that up-or-out prevails when firm-specific human capital is low and high- and low-level jobs are similar. Otherwise, standard (up-or-stay) practices are optimal. Similarly to our paper, Ghatak et al. (2001) study an overlapping

\footnotetext{
${ }^{6}$ Some actors and cast members have had very restrictive contracts. For example, George Reeves' (Superman in the 1950s) contract prevented him from taking other work that might interfere with the series by including a "30-day clause", which meant that the producers could demand his exclusive services for a new season on four weeks' notice. Similarly, publishers such as comic producers, may retain the creator of the comic book character by retaining copyrights associated with this character and the stories. These companies own the copyrights to the artist's entire professional portfolio and have exclusivity over representations of characters created by the artist. After leaving a publishing firm, an artist is typically required to create from scratch. Finally, managers, scientists, and engineers can be prohibited from working for a competitor, on the ground that they would inevitably disclose trade secrets (for an analysis of some consequences of the "doctrine of inevitable disclosure" see, for example, Png and Samila, 2013).
} 
generations version of a principal-agent problem in which contracts are determined in general equilibrium. In their model, all young workers are identical but, depending on their performance, will have different investment possibilities as seniors. Ghatak et al. (2001) do not allow for long-term contracts because their aim is to explain seniors' choices between becoming entrepreneurs or remaining workers.

Our paper is also related to the optimal assignment literature that follows Becker (1973), who presents a theory of marriage and shows that positive assortative matching arises when abilities (or any other characteristics) are productive complements. Anderson and Smith (2010) develop a dynamic model of the labour market where only nature knows the workers' and firms' types. However, there is learning in this model because agents can update their beliefs about each of the matched partners through observation of a joint public signal. In their model, productive complementarity no longer implies positive assortative matching. There is a conflict between productive and dynamic efficiency: less output today may lead to a more profitable measure over types tomorrow. Informational concerns dominate productive concerns for agents whose "reputation" (that is, their publicly observed odds of being high productivity types) is neither too high nor too low. Eeckhout and Weng (2010) follow this line of research and study a different set-up where firms' characteristics are publicly known. In their framework, where there is a one-sided inference problem, positive assortative matching always arises under productive complementarity. Moreover, in the previous two papers, the competitive equilibrium implements the planner's stationary solution that maximises the aggregate flow of output. ${ }^{7}$

We assume a production function where the value of a firm's project and workers' abilities are complements. Supermodularity is maintained after incorporation into the model of moral hazard, which implies that utility is not perfectly transferable between firms and workers. Therefore, the existence of positive sorting in short-term contracts follows from the characteristics of the production function and Becker's result. The new, interesting, conflict that appears in our model occurs between the benefits of sorting, which require the flexibility associated with short-term contracts, and the superior incentives provided by long-term contracts, which preclude sorting. That is, positive assortative matching does not occur for all the participants because it requires giving up the gains associated with long-term partnerships. Finally, the nature of this trade-off implies that

\footnotetext{
${ }^{7}$ Both papers study supermodular as well as submodular production functions.
} 
the competitive equilibrium does not necessarily lead to the socially efficient solution. In terms of total surplus, the market leads to overuse of long-term contracts.

The remainder of the paper is organised as follows. Section 2 presents the model. Sections 3, 4 and 5 analyse the candidates for long- and short-term contracts in equilibrium. Section 6 characterises the identity of the firms and workers that enter into the relationship, the equilibrium salaries, and the contracts that emerge as a result of market interactions. Section 7 discusses some implications of the model when the set of firms is endogenous. Finally, Section 8 concludes.

\section{Model}

We model the economy as an overlapping generation model where at each period $t$, with $t=1,2, \ldots$, firms contract with workers to develop projects. Firms are infinitely-lived players, and the set of firms is constant for all periods. On the other hand, workers (agents) live for two periods. Both firms and workers discount the future according to the discount factor $\delta$, where $\delta \in(0,1)$.

All participants are assumed to be risk neutral. We also assume that a worker, at any age, enjoys limited liability over income. We normalise the minimum wage to zero. Therefore, we assume that the wage in any period and contingency cannot be negative.

At any period $t$, each firm is endowed with a project. The revenue for the firm from the project is $F+R$, if it is developed successfully, and $F>0$ if it is not developed successfully (i.e., is a failure). $F$ can be interpreted as a fixed component of the revenue that is not subject to uncertainty. The additional value of the project in case of success, $R$, is public information; it is the same across periods for a given firm but varies across firms. It is distributed in the interval $[\underline{R}, \bar{R}]$, with $\underline{R}>0$, according to the distribution function $G(R)$. Hence, the set of firms can also be identified as the interval $[\underline{R}, \bar{R}]{ }^{8}$ We

\footnotetext{
${ }^{8}$ In our model, all firms have the same $F$, while they are heterogeneous with respect to $R$, so that a "good" firm is a firm with a high $R$. However, workers have no influence over $F$, and decisions do not depend on $F$. Therefore, our analysis is independent of whether $F$ is the same for all the firms or varies from one firm to another. We can assume an arbitrary function $F(R)$ that associates a fixed revenue $F$ to each variable revenue $R$, with all our basic results unchanched in this more general scenario. It could be the case, for example, that $F(R)$ is decreasing and that firms with a high $R$ have lower expected profits than firms with a low $R$.
} 
assume that the set of active firms $[\underline{R}, \bar{R}]$ and the distribution function $G(R)$ are given; we will discuss these assumptions in Section 7.

In each period, a generation of workers is born. We assume that the measure of the set of workers born in any period is larger than the measure of the set of firms. Therefore, in period $t$ the market is composed of the set of firms, the set of workers that enter the market during this period and the set of older workers that entered the market in period $t-1$. In period 1 , there is a set of workers who are already old.

To run its project, a firm must hire a non-trained worker and a trained worker. Any agent is a non-trained worker during the first period that he works in this market. After this first job, a young agent who has worked for a firm becomes a trained worker; that is, working for a firm gives the agent the necessary skills to take charge of a project. ${ }^{9}$ We will also refer to non-trained and trained workers as junior and senior agents, respectively. ${ }^{10}$ According to our assumptions, there are more junior workers than non-trained positions to fill in the market.

A senior agent enters a relationship only if his expected utility is at least equal to some "outside utility" that we denote by $\underline{U}$. That is, $\underline{U}$ is the level of utility that an agent can secure outside the economy, in any period, and can be understood as his utility outside the labour market. Similarly, a junior agent accepts a contract only if his expected intertemporal utility is at least $\underline{U}+\delta \underline{U}$.

All workers are indistinguishable when junior. However, when senior, agents may have different abilities. An agent's ability to run a project as a senior is ex-ante unknown to all players, including the agent himself. There is public learning of the ability once the agent has worked for any firm, that is, when he is senior.

A junior agent working for a firm performs a routine job and exerts a predetermined

\footnotetext{
${ }^{9}$ In our model, workers can develop their potential to run projects as seniors independently of the type of firm with which they are employed. If only the firms with the most profitable projects would allow the junior workers to eventually develop their abilities, then movements of workers from low- to high-profit firms would not occur and short-term contracts would lose their advantage.

${ }^{10}$ Those agents who did not work for any firm when young could also be hired as non-trained workers when old. In other words, the time spent working as a junior is both probational and formative. However, we will assume that they are no longer in the market. As will become clear once we develop our analysis, this is just a simplification, as no firm would prefer to hire an old-non-trained worker rather than a junior one.
} 
and contractible level of effort. ${ }^{11}$ We normalise the cost for the agent of exerting this effort to zero. If we denote by $w_{J}$ the payment to the junior agent, then his utility at this age is $w_{J}$.

A senior agent working for a firm runs the project and his effort level (or his decision) is crucial to its success. This specialised effort is not contractible (not verifiable), and it influences the probability of success of the project. We assume that the probability of success is $p e$, where $e$ is the senior agent's effort and the parameter $p$ summarises his ability. ${ }^{12}$ The ability $p$ can take two values: $p_{H}$ or $p_{L}$, with $p_{H}>p_{L}$; that is, for the same effort $e>0$, a high-ability senior agent has a higher probability of success $\left(p_{H} e\right)$ than a low-ability senior agent $\left(p_{L} e\right)$. As noted above, it is only after being employed as a junior worker that an agent's (industry-specific) ability is public knowledge. ${ }^{13}$ Ex-ante, there is a proportion $q$ of high-ability agents in the population.

The result of the project (success or failure) is verifiable; hence, the agents' payment can depend on it. Given that the junior agent's effort is verifiable, there is no reason to offer him a contingent payment, and a fixed salary $w_{J} \geq 0$ is optimal. On the other hand, a contingent payment scheme should be offered to the senior agent to incentivise his work effort. We denote the incentive contract by $C_{S}=\left(w_{S}, \Delta_{S}\right)$, where the first component, $w_{S} \geq 0$, is the base payment, i.e., the transfer in case of failure, whereas the second part, $\Delta_{S}$, is the bonus in case revenue $R$ is obtained. The contract offered may vary, depending on the (publicly known) ability of the senior worker, that is, we may have $C_{H}$ different from $C_{L}$. Given the contract $\left(w_{S}, \Delta_{S}\right)$, the expected utility of a senior agent of ability $p$ is

$$
w_{S}+p e \Delta_{S}-\frac{1}{2} e^{2}
$$

where $\frac{1}{2} e^{2}$ represents the cost of supplying effort $e$. Under contract $\left(w_{S}, \Delta_{S}\right)$, the senior agent selects the effort that maximises his expected utility, i.e.,

$$
e=\arg \max _{\widehat{e}}\left\{w_{S}+p \widehat{e} \Delta_{S}-\frac{1}{2} \widehat{e}^{2}\right\}
$$

\footnotetext{
${ }^{11}$ The assumption that the junior agent's effort is contractible enables a simple set-up. The main conclusions of our analysis carry over to a more complex model where the junior agent's effort is subject to moral hazard, provided the worker's effort does not affect the learning of his ability.

${ }^{12}$ We assume that the parameters of the model ensure that $p e$ is always smaller than 1.

${ }^{13}$ If there were some firm-specific human capital, workers who change jobs in the second period of their lives would lose their firm-specific formation.
} 
Therefore, the level of effort is

$$
e=p \Delta_{S}
$$

The previous equation represents the Incentive Compatibility Constraint $(I C C)$ of a senior agent. The agent tends to exert a higher level of effort, the larger is his bonus $\left(\Delta_{S}\right)$, and the higher are his skills $(p)$.

In any period $t$, the expected profits of an active firm that runs its project with a junior agent, to whom it pays the salary $w_{J}$, and a senior agent of ability $p$, who is paid according to the payment scheme $\left(w_{S}, \Delta_{S}\right)$, are

$$
F-w_{J}+p e\left(R-\Delta_{S}\right)-w_{S}
$$

Firms and workers in this market can sign either Short-term (ST) or Long-term (LT) contracts. A ST contract between a firm and a junior agent consists of a salary $w_{J}$. A ST contract between a firm and a senior agent is an incentive scheme $\left(w_{S}, \Delta_{S}\right)$ that may differ for agents with different abilities (and can also depend on the value of the project in the case of success). A senior agent had worked for some firm in period $t-1$ but not necessarily for the same firm with which he signs the ST contract at period $t$. A LT contract between a firm and a junior agent in period $t$ specifies the salary that the worker will receive during the first period of the relationship and the incentive scheme that will govern the relationship in period $t+1$. The incentive scheme can be a function of the revealed ability of the agent. That is, a LT contract is a vector $\left(w_{J}, w_{H}, \Delta_{H}, w_{L}, \Delta_{L}\right)$ that implies a commitment by the firm to retain the worker as a senior and a commitment by the agent to work for the same firm in period $t+1$, independently of his ability.

In each period, each firm determines the contracts it offers and the type of workers it hires. Therefore, an Outcome in the economy specifies, for each period $t$, the assignment of (some) junior and senior workers to firms and the contracts that govern their relationship. Recall that a contract between a firm and a junior agent in period $t$ is signed in this period and can be either ST or LT. On the other hand, the contract governing the relationship between a senior worker and a firm in period $t$ can be either a ST contract signed in this period or a LT contract signed in period $t-1$.

In any period $t$, the contracts signed involve, on the one hand, all firms and the junior workers, that may chose either a ST or LT contract and, on the other hand, firms and the senior workers who signed ST contracts in period $t-1$. We seek equilibrium outcomes, 
that is, outcomes that are immune to deviations in any period $t$. First, contracts must be individually rational for the agents and the firms. Any (junior or senior) worker who signs a contract in period $t$ must be better off under the proposed outcome than if he did not enter the relationship. Additionally, firms must obtain non-negative profits. For simplicity, we assume that all firms in $[\underline{R}, \bar{R}]$ are active in the market, that is, we assume that the firms' participation constraint holds. ${ }^{14}$ Second, a firm and any worker that sign a contract in period $t$ cannot be better off by signing a different contract than the one that obtains in the equilibrium outcome. This implies that any equilibrium contract that a firm-worker pair signs at $t$ cannot be improved. In addition, a firm and a (junior or senior) worker that are not matched at $t$, under the proposed equilibrium, cannot be made better off by signing a contract among themselves. Therefore, an equilibrium outcome is immune to deviations by any firm and any worker: the firm has no incentive to deviate from the proposed outcome by changing the contract with its assigned workers or by contracting with other agents. Note that a firm can keep or attract a worker in period $t$ if it offers him a contract under which he obtains the same level of utility (or a slightly higher level of utility) than his current level.

Hence, Equilibrium in the economy is an outcome where:

a) all active workers obtain at least their outside utility (i.e., junior workers achieve an expected total utility of at least $(1+\delta) \underline{U}$, and senior workers obtain an expected utility of at least $\underline{U}$, if they sign a contract at their respective ages);

b) no firm would obtain higher expected intertemporal profits by changing the set of proposed contracts to another set of contracts that guarantee to each worker at least the same level of expected utility as is obtained under the current outcome.

We focus the analysis on stationary equilibria, that is, on equilibria where firms follow the same strategy every period. ${ }^{15}$ For simplicity, we refer to stationary equilibria simply as equilibria.

In equilibrium, a firm may offer

- LT contracts to junior workers (that it retains them as seniors, whatever their type),

\footnotetext{
${ }^{14}$ We return to this issue in Section 7.

${ }^{15}$ Our model is stationary. In particular, firms do not face any liquidity constraint, so their decision in a certain period does not depend on the success or failure of the previous period's project. Otherwise, a firm might fluctuate between hiring a young agent through a LT or ST contract, depending on the past history of profits.
} 
or

- ST contracts to junior workers and to senior workers of high and low ability.

Therefore, in equilibrium the set of firms $[\underline{R}, \bar{R}]$ is partitioned into three subsets, some of which may be empty: the set $\mathcal{R}^{L T}$ of firms that offer LT contracts, the set $\mathcal{R}^{H}$ of firms that offer ST contracts to juniors and hire high-ability seniors and the set $\mathcal{R}^{L}$ of firms that offer ST contracts to juniors and hire low-ability seniors. Obviously, the measures of the sets $\mathcal{R}^{H}$ and $\mathcal{R}^{L}$ cannot be arbitrary as they must satisfy the feasibility constraint that the ratio of the measures of $\mathcal{R}^{H}$ and $\mathcal{R}^{L}$ must be equal to the ratio of high- to low-ability workers $\left(\frac{q}{1-q}\right)$.

We note first that when one considers the analysis of one isolated firm's optimal contract, ST contracts are always (at least weakly) dominated by LT contracts. We do not prove this result, as it is well established in the literature that LT contracts typically improve the efficiency of relationships by allowing both parties to commit to a sequence of events. ${ }^{16}$ The intuition is that the firm can always replicate in the LT contract the optimal sequence of contingent ST contracts. Moreover, LT contracts are typically superior because the firm, when it signs ST contracts, cannot commit to paying the senior worker a utility level above his reservation utility. Therefore, if the firm wishes to retain the senior agent independently of his type, it would obtain higher profits by signing a LT contract. In other words, we need not consider a fourth set of firms - those that sign ST contracts and re-hire the same worker as a senior independently of his type - as this set is always empty in equilibrium.

Proposition 1 uses this idea to show a stronger result: in our environment, a situation in which all firms sign (optimal) LT contracts with their workers is an equilibrium.

Proposition 1 There is always an equilibrium where all active firms sign LT contracts with their workers.

\footnotetext{
${ }^{16}$ On the optimality of LT contracts versus ST contracts in a single principal-agent model with repeated moral hazard, see, for instance, Lambert (1983), Rogerson (1985), Malcomson and Spinnewyn (1988), and Chiappori et al. (1994). This literature analyses the role of commitment, reputation, memory and renegotiation. In our set-up, the key element is commitment. There is no role for memory, as there is no past outcome, and there is no role for reputation, as all relevant parameters are public information at any time.
} 
If all the firms in the economy sign LT contracts, then no single firm has an incentive to deviate and offer a sequence of ST contracts. What is the advantage of committing to a LT relationship? As discussed above, the commitment allows the firm to relax the limited liability constraint of senior workers by delaying part of their payments as juniors. ST contracts typically cannot replicate this strategy. Indeed, when the firm designs a ST agreement for a senior agent, the contract that governed their relationship in the previous period, when the agent was young, is sunk. Therefore, the firm will only give the agent the rents that maximise its second-period profits.

The next sections explore equilibria where ST and LT contracts may coexist. We first discuss how to analyse equilibrium contracts by using a firm's one-period profits.

\section{Equilibria and one-period profits}

In equilibrium, no firm can obtain higher expected intertemporal profits by changing the set of proposed contracts. In this section, we relate this condition to the equilibrium profits that one firm obtains (or may obtain by changing the contract) in one period.

To illustrate the discussion, we take a firm offering at every period the LT contract $C^{L T}=\left(w_{J}, w_{H}, \Delta_{H}, w_{L}, \Delta_{L}\right)$. Because of the single-deviation principle, we consider a deviation by the firm from the contract it offers to the junior worker that it hires in a particular period $t$ (it maintains the offer $C^{L T}$ to agents born in any other period). If the firm deviates at $t$ to $C^{L T^{\prime}}=\left(w_{J}^{\prime}, w_{H}^{\prime}, \Delta_{H}^{\prime}, w_{L}^{\prime}, \Delta_{L}^{\prime}\right)$, then it pays $w_{J}^{\prime}$ at $t$ and offers $\left(w_{H}^{\prime}, \Delta_{H}^{\prime}, w_{L}^{\prime}, \Delta_{L}^{\prime}\right)$ in period $t+1$, where the change in income also takes place. Consequently, switching from $C^{L T}$ to $C^{L T \prime}$ in $t$ is not profitable for the firm if its profits at $t+1$ are not higher than under $C^{L T}$, taking into account that the change in the cost of the junior worker occurs at $t$. This is equivalent to comparing profits under $C^{L T}$ and under $C^{L T \prime}$, but considering the present value of the cost of the junior agent incurred in the previous period. That is, we impute a cost of $\frac{1}{\delta} w_{J}$ and $\frac{1}{\delta} w_{J}^{\prime}$ instead of using $w_{J}$ and $w_{J}^{\prime}$.

The situation is similar if a firm currently offering the contract $C^{L T}$ decides to offer the junior worker a ST contract at $t$ : the change in the cost of this worker occurs at $t$, whereas the change in the cost of the senior worker and in income occurs at $t+1$. Additionally, we face the same situation if a firm is currently offering ST contracts and 
plans to deviate to a LT contract at $t$ : it changes the agreement with the junior agent at $t$ to realize the new income at $t+1$. Finally, when a firm switches from a ST contract to another ST contract for the junior agent born at $t$, it hires the junior agent under the same conditions as before (that is, under the lowest salary that the agent is willing to accept) and changes his contract at $t+1$. Whether we compute the cost of the junior agent as $\frac{1}{\delta} w_{J}$ or $w_{J}$ is not relevant for the comparison of profits under the two strategies, as the firm pays the same cost under both, the old and new contracts.

Therefore, we can develop the analysis of the (stationary) equilibria of our model by focusing on the profits firms make in one period, provided that we view the cost of the junior agent as being in the previous period, that is, as long as we associate with the junior agent a cost of $\frac{1}{\delta} w_{J}$ rather than $w_{J}$. Henceforth, we will refer to this level of profits as "a firm's one-period profits" and where $E \tilde{\pi}=F-\frac{1}{\delta} w_{J}+p e\left(R-\Delta_{S}\right)-w_{S}$. A firm has incentives to switch from contract $C$ to contract $C^{\prime}$ if and only if $E \widetilde{\pi}(C)<E \widetilde{\pi}\left(C^{\prime}\right)$.

\section{Long-term contracts in equilibrium}

Consider a firm that owns a project $R \in[\underline{R}, \bar{R}]$ and that signs LT contracts with junior workers. At each period $t$, the firm runs the project with the junior agent that it hires in period $t$ and with the senior worker that it hired in period $t-1$. The senior agent has ability $p_{H}$ with probability $q$ and ability $p_{L}$ with probability $1-q$, as his ability was unknown at $t-1$. As previously noted, the ability of the agent is publicly known before he starts work as a senior; hence, the LT contract signed in $t-1$ may stipulate payments contingent on the ability of the agent as a senior.

All workers are ex-ante identical and there are more junior workers than positions to fill. Therefore, in any period, there are unemployed junior agents willing to accept any LT contract that provides them with an expected utility equal to their (two-period) outside utility $(1+\delta) \underline{U}$. Hence, the participation constraint $(P C)$ specifies that the total expected utility the worker obtains in the relationship must be at least $(1+\delta) \underline{U}$.

Following the discussion of the previous section, a candidate LT contract for equilibrium $\left(w_{J}, w_{H}, \Delta_{H}, w_{L}, \Delta_{L}\right)$ maximises the firm's one-period profits, also taking into account the incentive compatibility $(I C C \mathrm{~s})$ and limited liability constraints $(L L C)$. That 
is, it solves

$$
\begin{gathered}
\max _{\left(w_{J}, w_{H}, \Delta_{H}, w_{L}, \Delta_{L}\right)} F-\frac{1}{\delta} w_{J}+q\left(p_{H} e_{H}\left(R-\Delta_{H}\right)-w_{H}\right)+(1-q)\left(p_{L} e_{L}\left(R-\Delta_{L}\right)-w_{L}\right) \\
\text { s.t. } w_{J}+\delta\left[q\left(p_{H} e_{H} \Delta_{H}+w_{H}-\frac{1}{2} e_{H}^{2}\right)+(1-q)\left(p_{L} e_{L} \Delta_{L}+w_{L}-\frac{1}{2} e_{L}^{2}\right)\right] \geq(1+\delta) \underline{U} \\
e_{H}=p_{H} \Delta_{H}, \quad e_{L}=p_{L} \Delta_{L}, \quad w_{J} \geq 0, \quad w_{H} \geq 0, \quad w_{L} \geq 0 .
\end{gathered}
$$

If the contract does not satisfy this program, then the firm can deviate by offering a different acceptable LT agreement to junior agents and obtain larger discounted profits. We state the characteristics of the candidate LT contract in Proposition 2, where $\underline{\underline{U}} \equiv$ $\frac{1}{\delta}(1+\delta) \underline{U}$ and $\widetilde{p} \equiv \sqrt{q p_{H}^{2}+(1-q) p_{L}^{2}}$

Proposition 2 If firm $R$ is in the set $\mathcal{R}^{L T}$, then it offers the following LT contract:

Region $a^{L T}$ : If $R<\frac{1}{\widetilde{p}} \sqrt{2 \underline{\underline{U}}}$, then ${ }^{17}$

$$
C^{L T}(R)=\left(w_{J}^{L T}=0, w_{H}^{L T}=w_{L}^{L T}=\underline{\underline{U}}-\frac{1}{2} R^{2} \widetilde{p}^{2}, \Delta_{H}^{L T}=\Delta_{L}^{L T}=R\right) .
$$

Region $b^{L T}:$ If $R \in\left[\frac{1}{\widetilde{p}} \sqrt{2 \underline{\underline{U}}}, \frac{2}{\widetilde{p}} \sqrt{2 \underline{\underline{U}}}\right]$, then

$$
C^{L T}(R)=\left(w_{J}^{L T}=0, w_{H}^{L T}=w_{L}^{L T}=0, \Delta_{H}^{L T}=\Delta_{L}^{L T}=\frac{1}{\widetilde{p}} \sqrt{2 \underline{\underline{U}}}\right) .
$$

Region $c^{L T}$ : If $R>\frac{2}{\widetilde{p}} \sqrt{2 \underline{\underline{U}}}$, then

$$
C^{L T}(R)=\left(w_{J}^{L T}=0, w_{H}^{L T}=w_{L}^{L T}=0, \Delta_{H}^{L T}=\Delta_{L}^{L T}=\frac{R}{2}\right) .
$$

We now explain the main characteristics of the LT contract $C^{L T}(R)$. Owing to moral hazard arising from limited liability, the firm's capacity to induce the senior worker to exert a high effort level is restricted. Therefore, the firm desires to relax the senior agent's limited liability constraint, which explains why it concentrates as much as possible of the agent's compensation in the agent's second period of life (i.e., the firm pays to a young worker the minimum possible wage: $w_{J}^{L T}=0$.) Young agents accept low wages because

\footnotetext{
${ }^{17}$ In this region, there are other contracts that are also candidates for equilibrium. Because agents exert first-best effort and there is slack in the LLC constraint, flat wage payments can be shifted across periods and states while keeping payoffs the same for both the agent and the firm. Any combination of $w_{J}, w_{H}$ and $w_{L}$ that satisfies $w_{J}+\delta\left(q w_{H}+(1-q) w_{L}\right)+\delta \frac{1}{2} R^{2} \widetilde{p}^{2}=(1+\delta) \underline{U}$, such that each variable is non-negative, is also a candidate equilibrium, as it would give the same profits to the firm.
} 


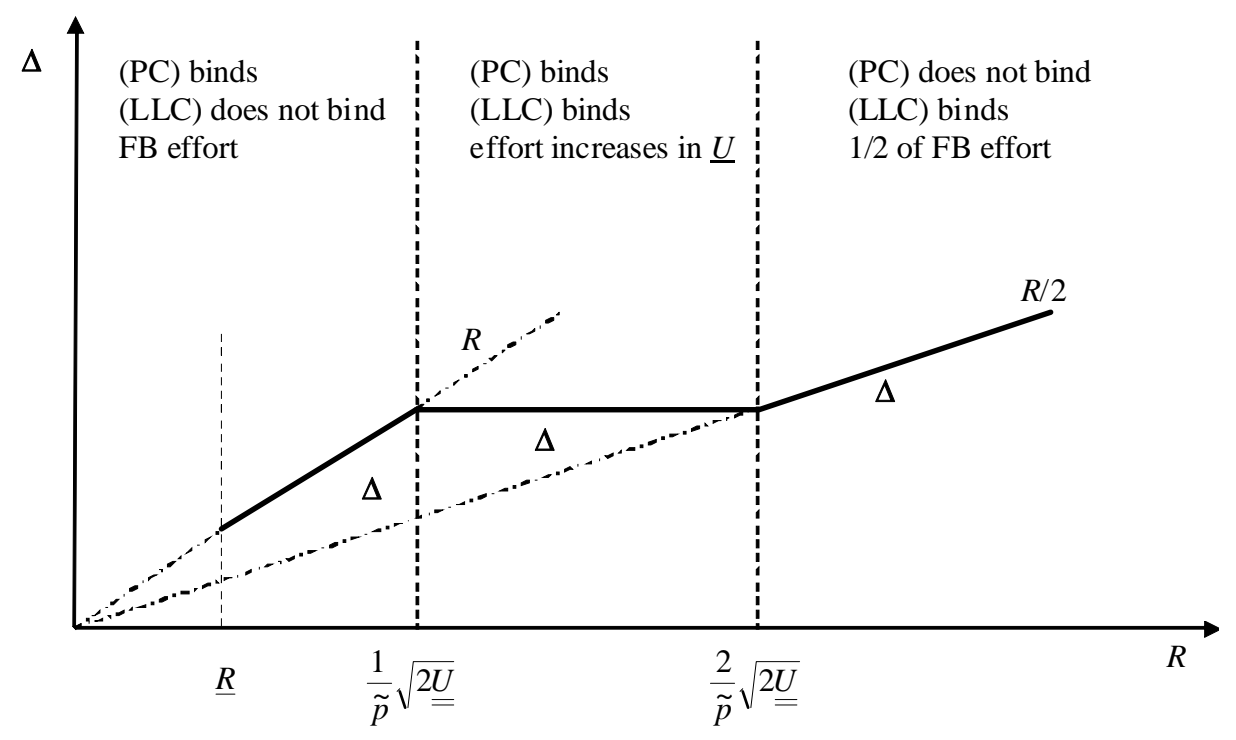

Figure 1: Incentives in the optimal LT contracts

of the credible promise to be "well" paid when they are senior. The limited liability constraints also explain why, unless $R$ is very low, workers are paid the minimum salary if the outcome turns out to be a failure: $w_{H}^{L T}=w_{L}^{L T}=0$.

The impact of limited liability on bonuses and payoffs obtained by agents and firms differs depending on the profitability of the project $R$ (as well as on the level of agents' reservation utility $(1+\delta) \underline{U}$ and "average" probability of success $\widetilde{p})$. Some characteristics of the optimal LT contract are shown in Figure 1.

To induce the agent's first-best effort, firm $R$ must offer him a bonus of $\Delta=R$. If the value of the project is low (Region $a^{L T}$ ), the first-best effort-inducing bonus payment is less than the agent's outside option. Therefore, the LLC does not bind. Utility is transferable in this region, so the contract induces the first-best effort $\left(e_{i}^{L T}=p_{i} R\right.$, for $i=H, L)$ and accompanies these incentives with a transfer payment to match the agent's outside option $\left(w_{H}^{L T}=w_{L}^{L T}>0\right)$.

For $R \geq \frac{1}{\widetilde{p}} \sqrt{2 \underline{\underline{U}}}$, the bonus $\Delta=R$ ensures the agent a utility larger than his outside option, and the LLC binds. In this case, firm $R$ may pay the agent more than his reservation utility and/or reduce the effort below the first-best level. Firms with intermediate values of $R$ (Region $b^{L T}$ ) prefer paying the minimum bonus $\left(\Delta=\frac{1}{\widetilde{p}} \sqrt{2 \underline{\underline{U}}}\right.$ ) that gives the agent his outside option rather than making an additional transfer to the agent. In 
this region, both the LLC and the PC are binding, the contract does not include fixed payments and the senior agent's effort is $e_{i}^{L T}=\frac{p_{i}}{\widetilde{p}} \sqrt{2 \underline{\underline{U}}}$, for $i=H, L$.

In Region $c^{L T}$, the value of $R$ is sufficiently high that firms choose to increase the bonus beyond that given by the PC. In this region, bonuses are no longer influenced by the PC but by the LLC. Given the firm's cost of transferring rents to agents, the firm, at the optimum, shares half of the value of the project with the senior agent in the event of success $\left(\Delta=\frac{R}{2}\right)$ to induce the senior worker to supply effort $e_{i}^{L T}=\frac{1}{2} p_{i} R$, for $i=H, L$. Thus, the agent obtains rents in addition to his outside option.

The next corollary provides the expression of the firm's one-period profits for $C^{L T}(R)$.

Corollary 1 The firm's one-period profits under $C^{L T}(R)$ are:

Region $a^{L T}$ : If $R<\frac{1}{\widetilde{p}} \sqrt{2 \underline{\underline{U}}}$, then $E \widetilde{\pi}^{L T}(R)=F-\underline{\underline{U}}+\frac{1}{2} R^{2} \widetilde{p}^{2}$.

Region $b^{L T}:$ If $R \in\left[\frac{1}{\widetilde{p}} \sqrt{2 \underline{\underline{U}}}, \frac{2}{\widetilde{p}} \sqrt{2 \underline{\underline{U}}}\right]$, then $E \widetilde{\pi}^{L T}(R)=F+R \widetilde{p} \sqrt{2 \underline{\underline{U}}}-2 \underline{\underline{U}}$.

Region $c^{L T}$ : If $R>\frac{2}{\widetilde{p}} \sqrt{2 \underline{\underline{U}}}$, then $E \widetilde{\pi}^{L T}(R)=F+\frac{1}{4} R^{2} \widetilde{p}^{2}$.

The profit function $E \widetilde{\pi}^{L T}(R)$ is continuously differentiable and convex in $R$.

\section{Short-term contracts in equilibrium}

All firms signing ST contracts hire similar young workers, as they are indistinguishable ex-ante. With respect to senior workers, firms can decide to hire high-ability or low-ability workers.

Consider an equilibrium where some firms sign ST contracts, with a fraction of those firms offering contracts to high-ability senior agents. Denote by $U_{H}$ the (minimum) level of utility that this type of agent obtains in equilibrium. ${ }^{18}$ Similarly, denote by $U_{L}$ the (minimum) level of utility obtained by low-ability senior workers. Both $U_{H}$ and $U_{L}$ exceed or equal $\underline{U}$. Additionally, given the limited liability constraint and competition among firms, $U_{H}$ and possibly $U_{L}$ may strictly exceed $\underline{U}$. Therefore, a junior agent will sign a ST contract that provides a utility level less than $\underline{U}$ provided that the reduction does not exceed the expected extra utility he will obtain as a senior. Formally, the salary $w_{J}$ that

\footnotetext{
${ }^{18}$ Given the limited liability constraint, the participation constraint may not be relevant. Therefore, the equilibrium level of utility may differ for identical senior agents, depending on the firm that hires them. A firm with a very high $R$ ends up providing its senior agent a utility level higher than $U_{H}$, as its participation constraint will not be binding (see also Alonso-Paulí and Pérez-Castrillo, 2012).
} 
the junior agent will accept must satisfy:

$$
w_{J}+\delta\left[q E_{H}+(1-q) E_{L}\right] \geq(1+\delta) \underline{U},
$$

where $E_{H}$ and $E_{L}$ are the expected utilities of high- and low-ability workers, respectively. For example, if all low-ability workers obtain the same utility $U_{L}$ in the possible jobs, then $E_{L}=U_{L}$.

The candidate equilibrium contract offer of firm $R$ in $\mathcal{R}^{H}\left(\mathcal{R}^{L}\right)$ to a high- (low-) ability senior agent must be the optimal one-period contract for this agent, taking into account that it must grant him a level of utility of at least $U_{H}\left(U_{L}\right)$; that is, it must solve

$$
\begin{gathered}
\max _{\left(w_{i}, \Delta_{i}\right)} F+p_{i} e_{i}\left(R-\Delta_{i}\right)-w_{i} \\
\text { s.t. } \quad p_{i} e_{i} \Delta_{i}+w_{i}-\frac{1}{2} e_{i}^{2} \geq U_{i} \\
e_{i}=p_{i} \Delta_{i} \\
w_{i} \geq 0
\end{gathered}
$$

for $i=H, L$. The next proposition states the candidate equilibrium contract for those firms.

Proposition 3 If firm $R$ is in the set $\mathcal{R}^{i}$, with $i \in\{H, L\}$, then it offers the following $S T$ contract to a senior agent:

Region $a_{i}^{S T}\left(U_{i}\right):$ If $R<\frac{1}{p_{i}} \sqrt{2 U_{i}}$, then

$$
C_{i}^{S T}\left(R, U_{i}\right)=\left(w_{i}^{S T}=U_{i}-\frac{1}{2} p_{i}^{2} R^{2}, \Delta_{i}^{S T}=R\right)
$$

Region $b_{i}^{S T}\left(U_{i}\right):$ If $R \in\left[\frac{1}{p_{i}} \sqrt{2 U_{i}}, \frac{2}{p_{i}} \sqrt{2 U_{i}}\right]$, then

$$
C_{i}^{S T}\left(R, U_{i}\right)=\left(w_{i}^{S T}=0, \Delta_{i}^{S T}=\frac{1}{p_{i}} \sqrt{2 U_{i}}\right) .
$$

Region $c_{i}^{S T}\left(U_{i}\right):$ If $R>\frac{2}{p_{i}} \sqrt{2 U_{i}}$, then

$$
C_{i}^{S T}\left(R, U_{i}\right)=\left(w_{i}^{S T}=0, \Delta_{i}^{S T}=\frac{R}{2}\right)
$$

In Region $a_{i}^{S T}\left(U_{i}\right)$, a senior agent's effort is the first-best level effort $e_{i}^{S T}=p_{i} R$, whereas in Region $b_{i}^{S T}\left(U_{i}\right)$, his effort is less than the first-best level: $e_{i}^{S T}=\sqrt{2 U_{i}}$. In 
these two regions, the agent's expected utility is $U_{i}$. Finally, in Region $c_{i}^{S T}\left(U_{i}\right)$, where the project value is very high, the senior agent's effort is $e_{i}^{S T}=\frac{1}{2} p_{i} R$, for $i=H, L$, and he receives a rent in addition to $U_{i}$. His expected utility in this region is $\frac{1}{8} p_{i}^{2} R^{2}>U_{i}$.

Corollary 2 states the expression for the firm's one-period profits under $C_{i}^{S T}\left(R, U_{i}\right)$, where $w_{J}^{S T}$ denotes the equilibrium salary paid to junior agents.

Corollary 2 A firm $R$ in the set $\mathcal{R}^{i}$, with $i \in\{H, L\}$, obtains the following one-period profits under $C_{i}^{S T}\left(R, U_{i}\right)$

Region $a_{i}^{S T}\left(U_{i}\right)$ : If $R<\frac{1}{p_{i}} \sqrt{2 U_{i}}$, then $E \tilde{\pi}_{i}^{S T}\left(R, w_{J}^{S T}, U_{i}\right)=F+\frac{1}{2} p_{i}^{2} R^{2}-U_{i}-\frac{1}{\delta} w_{J}^{S T}$. Region $b_{i}^{S T}\left(U_{i}\right):$ If $R \in\left[\frac{1}{p_{i}} \sqrt{2 U_{i}}, \frac{2}{p_{i}} \sqrt{2 U_{i}}\right]$, then

$$
E \widetilde{\pi}_{i}^{S T}\left(R, w_{J}^{S T}, U_{i}\right)=F-2 U_{i}+p_{i} R \sqrt{2 U_{i}}-\frac{1}{\delta} w_{J}^{S T} .
$$

Region $c_{i}^{S T}\left(U_{i}\right)$ : If $R>\frac{2}{p_{i}} \sqrt{2 U_{i}}$, then $E \tilde{\pi}_{i}^{S T}\left(R, w_{J}^{S T}, U_{i}\right)=F+\frac{1}{4} p_{i}^{2} R^{2}-\frac{1}{\delta} w_{J}^{S T}$.

The profit function $E \widetilde{\pi}_{i}^{S T}\left(R, w_{J}^{S T}, U_{i}\right)$ is continuously differentiable and convex in $R$.

\section{Equilibrium matching and equilibrium contracts}

The previous sections identify the equilibrium contracts, once we know the type of agreements firms offer (that is, once the sets $\mathcal{R}^{L T}, \mathcal{R}^{H}$ and $\mathcal{R}^{L}$ are determined) and the levels of utility $U_{L}$ and $U_{H}$ that they must guarantee to low- and high-ability agents, respectively. In the present section, we characterise equilibria where at least some firms offer ST contracts. Therefore, we identify the distribution of firms in $\mathcal{R}^{L T}, \mathcal{R}^{H}$ and $\mathcal{R}^{L}$, the levels $U_{L}$ and $U_{H}$ and the minimum salary $w_{J}$ that firms offer junior agents in ST contracts.

We seek equilibria where $U_{L}=\underline{U}$. Firms will not compete for low-ability workers. ${ }^{19}$ On the other hand, the level of $U_{H}$ will be determined by the equilibrium conditions, that is, by the (marginal) firm's willingness to pay to attract a high-ability worker rather than either attracting a low-ability one, or signing a LT contract.

Why might some firms be interested in LT relationships while others prefer to secure high-ability agents through ST contracts? In particular, why would a firm choose a strategy that implies contracting low-ability agents through ST contracts, instead of offering LT contracts and sometimes benefiting from high-ability senior agents? The two main equilibrium variables that cause firms to prefer one or another type of contract are the

\footnotetext{
${ }^{19}$ However, in equilibrium, the measure of senior workers with low ability is the same as the measure of firms seeking them. Therefore, other equilibria may exist where $U_{L}>\underline{U}$ for all low-ability players.
} 
salary of a young worker $w_{J}^{S T}$ (or rather the comparison between $w_{J}^{S T}$ and $\underline{U}$ ) and the difference between the cost of a high- versus a low-ability senior agent, that is, $U_{H}-U_{L}$.

Firms that obtain large profits in the event of success, that is, firms with a high $R$, are willing to pay a high price to always hire a good senior agent. Therefore, firms at the right end of the interval $[\underline{R}, \bar{R}]$ must be those most interested in signing ST contracts with high-ability senior agents. Similarly, firms that care little about agents' efforts, i.e., firms with a low $R$, pay more attention to the potential savings they can obtain through a junior's contract if they offer him a ST contract than to the gains obtained through a LT contract or by securing a high-ability agent. Therefore, firms at the left end of $[\underline{R}, \bar{R}]$ are candidates to sign ST contracts to hire low-ability senior agents.

Lemma 1 provides the first confirmation of these intuitions. It compares the slopes, in terms of $R$, of the profits obtained from the different types of contract.

Lemma 1 The slopes of the profit functions satisfy the following relations:

(a) $\frac{\partial E \widetilde{\pi}_{L}^{S T}}{\partial R}\left(R, w_{J}, \underline{U}\right)<\frac{\partial E \tilde{\pi}^{L T}}{\partial R}(R)$, for all $R, w_{J}$;

(b) $\frac{\partial E \tilde{\pi}_{L}^{S T}}{\partial R}\left(R, w_{J}, \underline{U}\right)<\frac{\partial E \tilde{\pi}_{H}^{S T}}{\partial R}\left(R, w_{J}, U_{H}\right)$, for all $R, w_{J}$, and for all $U_{H} \geq \underline{U}$; and

(c) $\frac{\partial E \widetilde{\pi}^{L T}}{\partial R}(R)<\frac{\partial E \widetilde{\pi}_{H}^{S T}}{\partial R}\left(R, w_{J}, U_{H}\right)$, for all $R, w_{J}$, if either $U_{H}>\frac{\widetilde{p}^{2}}{p_{H}^{2}} \underline{\underline{U}}$ or $2 \widetilde{p}^{2}<p_{H}^{2}$.

A firm's ST profits increase with the value of success $R$ when it hires a low-ability worker. However, this increase is smaller than that of a firm's profits under the optimal LT contract (part (a)). It is also smaller than the rate at which its profits increase if it hires a high-ability worker through a ST contract (part (b)). These relations are explained by the fact that a higher $R$ implies a greater interest in securing the services of a high-ability worker. A similar argument provides the intuition for part (c) of the lemma.

Let us denote by $\widehat{R}$ the value that would "balance" the set of firms if all the firms with $R<\widehat{R}$ hired low-ability workers, while all the firms with $R \geq \widehat{R}$ hired high-ability workers, that is, $\widehat{R}$ is characterised by

$$
G(\widehat{R})=1-q
$$

Additionally, we denote by $R^{o}$ the value that makes the firm indifferent between using LT contracts and hiring low-ability senior workers through ST contracts, when the junior salary is $w_{J}^{S T}=0$. That is, $R^{o}$ is characterised by

$$
E \widetilde{\pi}^{L T}\left(R^{o}\right)=E \widetilde{\pi}_{L}^{S T}\left(R^{o}, 0, \underline{U}\right)
$$


Theorem 1 states an equilibrium when $R^{o} \in[\underline{R}, \widehat{R})$, that is, where some of the firms in the market have a low-valued project, but there is a relatively high number of firms with valuable projects. The theorem shows that, in this case, ST and LT contracts coexist in the market. Theorem 1 presents the result in the case where $p_{H}^{2}>\left(1+\frac{1}{\delta q}\right) p_{L}^{2}$, that is, $p_{H}$ is sufficiently high with respect to $p_{L}$. This hypothesis allows us to describe the equilibrium in a simple manner. However, as we will discuss at the end of this section, the coexistence of the two types of contract does not depend on this assumption.

Theorem 1 Suppose $\underline{R} \leq R^{o}<\widehat{R}$, and denote by $R^{o o}$ the firm such that $q G\left(R^{o}\right)=$ $(1-q)\left(1-G\left(R^{o o}\right)\right)$. Then, if $p_{H}^{2}>\left(1+\frac{1}{\delta q}\right) p_{L}^{2}$, an equilibrium exists where

(i) firms with $R \leq R^{o}$ offer $S T$ contracts: $w_{J}^{S T}=0$ to junior workers and $C_{L}^{S T}(R, \underline{U})$ to low-ability senior workers,

(ii) firms with $R \in\left(R^{o}, R^{o o}\right)$ offer LT contracts $C^{L T}(R)$,

(iii) firms with $R \geq R^{o o}$ offer $S T$ contracts: $w_{J}^{S T}=0$ to junior workers and $C_{H}^{S T}\left(R, U_{H}^{o o}\right)$ to high-ability senior workers, where $U_{H}^{o o}$ is such that $E \widetilde{\pi}^{L T}\left(R^{o o}\right)=E \widetilde{\pi}_{H}^{S T}\left(R^{o o}, 0, U_{H}^{o o}\right)$,

(iv) junior workers accept both LT contracts that guarantee them $(1+\delta) \underline{U}$ and $S T$ contracts with $w_{J}^{S T}=0$, and

(v) senior workers accept contracts that guarantee them $\underline{U} \cdot{ }^{20}$

The rationale behind Theorem 1 is as follows. Firms with more profitable projects attach greater importance to hiring high-ability workers. They can ensure the services of these workers by signing ST contracts and offering a high salary to attract senior agents of high ability. Therefore, the expected utility $U_{H}^{o o}$ obtained by agents who sign ST contracts and turn out to be high-ability senior workers is considerably higher than their outside option. This prospect increases the expected utility of junior workers who accept ST contracts: if they turn out to be of high ability they obtain a high utility level. The expectation of this potential reward leads junior workers to accept a wage of $w_{J}^{S T}=0$, which is less than their reservation utility $\underline{U}$ because they will be compensated in the future (in terms of expected utility) for this sacrifice. Firms with low $R$ take advantage of the reduction in the wage offered to junior workers who sign ST contracts: the value of their projects is sufficiently low so that the reduction in the wage of junior agents more than offsets the fact that they always end up hiring low-ability senior workers.

\footnotetext{
${ }^{20}$ At equilibrium, high-ability workers receive a level of utility of at least $U_{H}^{o o}>\underline{U}$. However, out of equilibrium, they should be ready to accept lower offers, as long as they guarantee $\underline{U}$.
} 
The firms with the best projects sign ST contracts because they are willing to pay a high premium to ensure the services of high-ability workers, whereas the firms with the worst projects sign ST contracts to benefit from the low salaries of junior workers. Firms with intermediate $R$ find high-ability workers to be too expensive. However, they benefit enough from the ability of such workers that the reduction in the salary of junior workers does not compensate for the loss they suffer by giving up the possibility of retaining highability workers. Therefore, they commit to LT contracts and benefit from the advantages arising from this type of agreement.

Theorem 1 shows positive assortative matching between senior workers and firms but only for firms with the best and worst projects. First, the property that high-ability agents work for firms with higher-value projects follows from our choice of production function, which yields a match-value function of $F+p e R-\frac{1}{2} e^{2}$ that is supermodular. In addition, supermodularity is preserved when we take into account the moral hazard problem and the firm's optimal choice of incentive scheme, which depends on the project value. Therefore, in the regions where the market for senior agents exists, the matching is positive assortative. Second, the theorem also highlights that the market for senior agents does not exist for firms with intermediate projects. They enter into LT contracts and remain matched even after new information about types is revealed. Similarly to the "coarse matching" literature, the coexistence of LT and ST contracts demonstrates that the trade-off between efficient contracting and efficient sorting need not be all-ornothing. ${ }^{21}$

Figure 2 indicates LT and ST profits as a function of $R$, for equilibrium values of salaries and utility $U_{H}$. In the figure, we represent the functions for the following parameters: $\delta=0.9, p_{H}=0.2, p_{L}=0.1, q=0.5, \underline{U}=0.05, F=1$, and where $G(R)$ is the uniform distribution function in $[0.5,8.5]$. As shown in Lemma 1 , the slope of $E \widetilde{\pi}_{H}^{S T}\left(R, 0, U_{H}^{o o}\right)$ is larger (because $U_{H}^{o o}$ is sufficiently high) than that of $E \widetilde{\pi}^{L T}(R)$, which in turn is larger than the slope of $E \widetilde{\pi}_{L}^{S T}(R, 0, \underline{U})$. In equilibrium, the market price that a firm must pay to attract a high-ability worker $\left(U_{H}^{o o}\right)$ is such that the three profit functions cross, as shown in Figure 2.

\footnotetext{
${ }^{21}$ Coarse matching provides a middle ground between perfect sorting and random matching. For example, under coarse matching types might sort into group buckets, but match randomly within the buckets (see, for example, Chao and Wilson, 1987, and Hoppe, Moldovanu and Ozdenoren, 2011).
} 


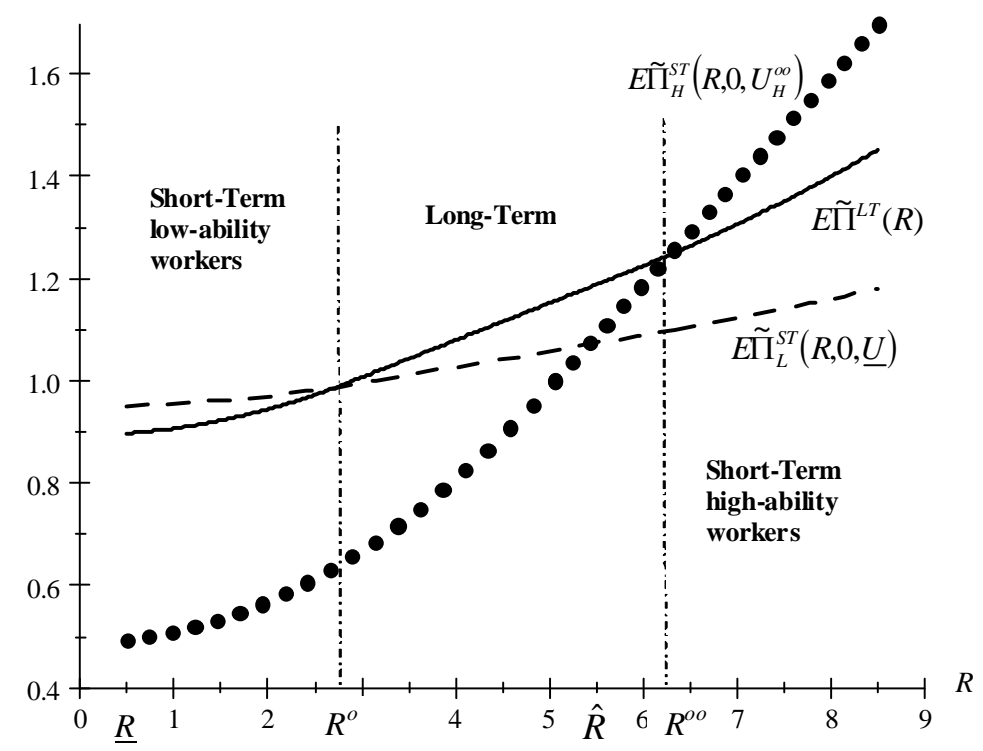

Figure 2: Profit Functions

Expected long-term profits are a convex combination of profits of the two possible realisations of agent type. In Figure 3, we decompose the LT profits function into the profits obtained through a LT contract when the agent turns out to be of type $H, E \widetilde{\pi}_{H}^{L T}(R)$ (in circles) and the profits when he turns out to be of type $L, E \widetilde{\pi}_{L}^{L T}(R)$ (in boxes). Note in particular that profits are higher under a LT contract when the agent turns out to be of high-ability than under a ST contract for sufficiently high values of $R .^{22}$

The previous numerical example also enables us to show that ST contracts favour ability. The expected utility of some high-ability seniors is much higher (and that of some seniors of low ability is lower) when LT and ST contracts coexist in equilibrium than when there are only LT contracts. At the right end of Figure 4, we observe a significant increase in the expected utility of high ability agents who sign ST contracts with very productive firms (high $R \mathrm{~s}$ ) compared to the utility they would have obtained with LT contracts. Conversely, the use of ST contracts in equilibrium makes low-ability workers worse-off than under LT contracts (left-hand side of Figure 4). Furthermore, if we view the population of firms as an industry composed of businesses of varying quality, our model

\footnotetext{
${ }^{22}$ Although it is difficult to see it in the figure, in Region $b$ profits for the LT contracts when the agent happens to be of type $L$ are lower than profits for the ST contracts with workers of type $L$.
} 


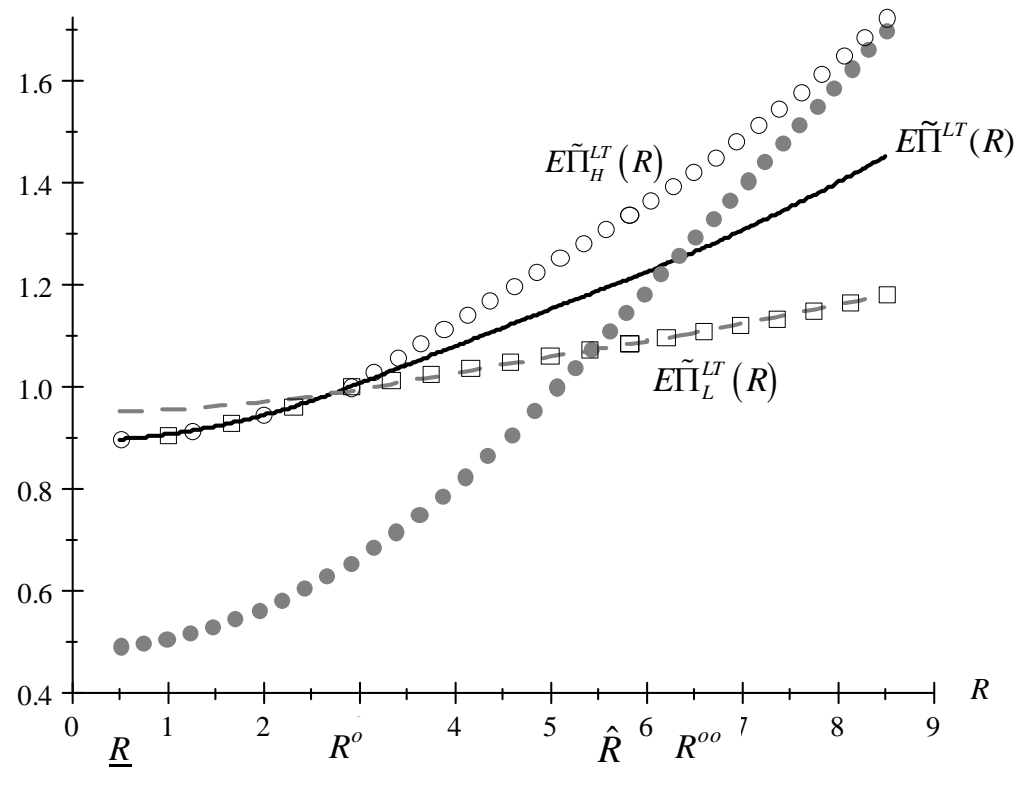

Figure 3: Profits with LT contracts depending on the type of the senior worker

provides an explanation for the observation that some firms reward their employees with higher wages than others, even when employees are otherwise identical (see, for example, Katz and Autor, 1999). High-ability seniors working in firms with moderately profitable projects have signed LT contracts and end up receiving compensations determined by their reservation utility, whereas the compensation of those who sign ST contracts is determined by competition. The latter end up working for high profit firms, supply more effort, and obtain higher utility. In contrast, low productivity workers in permanent jobs at moderately profitable firms receive higher payments as seniors and provide higher effort than their equals who signed ST contracts.

It is interesting to compare, in terms of efficiency, the equilibria obtained in Theorem 1 and in Proposition 1, where all firms sign LT contracts. On the one hand, we check that the equilibrium stated in Theorem 1 is preferred by firms to that in Proposition 1 . First, firms' profits under LT contracts are the same in both scenarios, as junior workers' outside option is the same in both cases. Second, firms that choose ST contracts in the equilibrium stated in Theorem 1 could have chosen LT contracts; therefore, their profits are higher under ST agreements (they are strictly higher in the interior of the regions). Third, a LT contract is certainly the best choice for any firm in the equilibrium described 


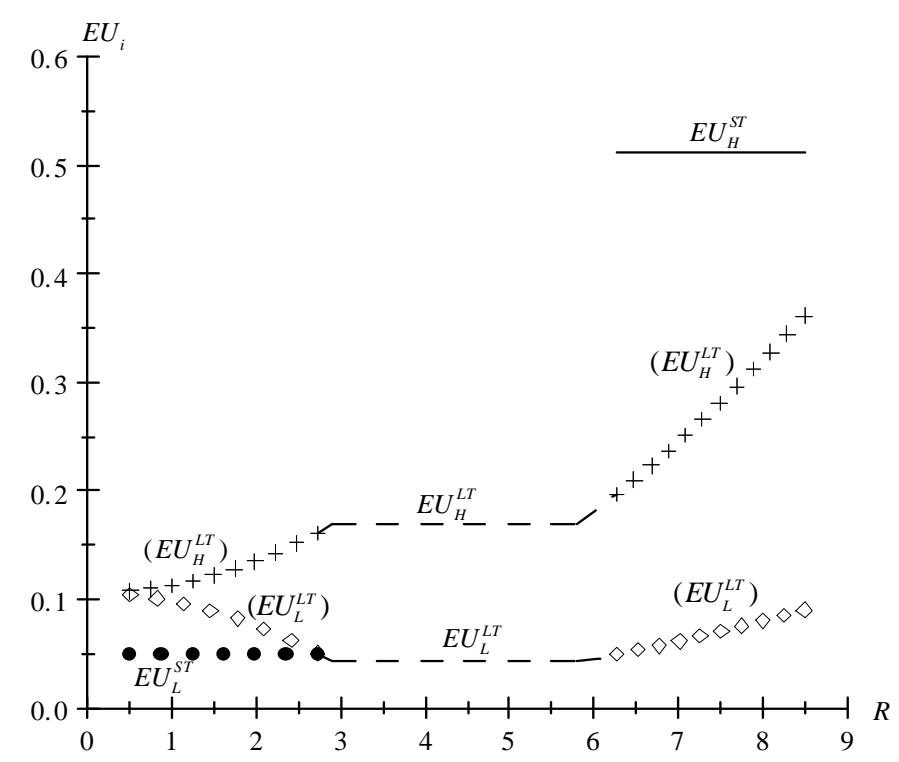

Figure 4: Expected utility of a senior agent of type $L$ and $H$ when he signs the equilibrium contract with a firm $R$. In parentheses, the expected utility if he had signed a LT contract.

in Proposition 1, as there is no market for high-ability and low-ability senior workers (if all the other senior workers are hired through LT contracts, the only possibility for the firm would be to retain its worker if it were to sign a ST agreement). Therefore, the equilibrium in Theorem 1 gives the firms a broader choice of contracts, and they are better-off.

On the other hand, the comparison between the two equilibria is not immediate for the workers. Although all junior workers are indistinguishable when they sign their equilibrium contracts, and they perform identical jobs, their expected utility varies depending on the type of agreement they are offered. Under a LT contract, a junior worker expects a total utility of $(1+\delta) \underline{U}$ if the firm's project $R$ is not too high. However, if $R$ is high $\left(R>\frac{2}{\widetilde{p}} \sqrt{2 \underline{\underline{U}}}\right)$, then his expected utility exceeds $(1+\delta) \underline{U}$ because the firm decides to increase his work incentives through an additional rent. Moreover, the expected utility of any worker who signs a ST contract with any firm is at least $\delta\left[q U_{H}^{o o}+(1-q) \underline{U}\right]$, which is strictly higher than $(1+\delta) \underline{U}$.

The next proposition shows that, under the assumptions in Theorem 1, workers are also better-off when ST and LT contracts coexist. The additional utility (in excess of the outside option) obtained by workers is larger when ST contracts are offered. It also shows 
that, in terms of total efficiency, the set of firms that sign ST contracts is still too small.

Proposition 4 Suppose $\underline{R} \leq R^{o}<\widehat{R}$ and $p_{H}^{2}>\left(1+\frac{1}{\delta q}\right) p_{L}^{2}$. Then

(i) the equilibrium with coexistence of LT and ST contracts proposed in Theorem 1 is preferred by firms and workers to the equilibrium proposed in Proposition 1; and

(ii) given $U_{H}^{o o}$, total welfare increases when the set of firms using ST contracts in the equilibrium proposed in Theorem 1 is marginally enlarged.

To briefly discuss the efficiency properties of the equilibrium with coexistence of LT and ST contracts, note that one possible interpretation of our model is that it analyses the trade-off between moral hazard and the optimal assignment of firms and workers. In this respect, it is useful to first examine the two extremes. On the one hand, if all workers were identical, then any assignment would be optimal and LT contracts would produce a higher total surplus than ST contracts because they alleviate the moral hazard problem. In addition, in equilibrium, we would only observe LT contracts. On the other hand, if there were no moral hazard problem (i.e., if effort were verifiable) then ST contracts would be efficient because they allow for the optimal assignment of firms and agents. In this case, an equilibrium in which all firms sign ST contracts (and high-ability senior workers receive a level of utility $\widehat{U}_{H}$ characterised by $\left.E \widetilde{\pi}^{L T}(\widehat{R})=E \widetilde{\pi}_{H}^{S T}\left(\widehat{R}, 0, \widehat{U}_{H}\right)\right)$ exists. Therefore, in the two extreme environments, the equilibrium assignment and contract length are socially optimal. Proposition 4 states that when there is a trade-off between moral hazard and optimal assignment, first, it is socially beneficial that some firms hire through ST contracts in equilibrium (part (i)) but, second, the equilibrium is not socially efficient: the market leads to overuse of LT contracts (part (ii)). The latter result contrasts with the equivalence between the competitive equilibrium and the planner's solution when the trade-off is between productive and informational efficiency (see Anderson and Smith, 2010, and Eeckhout and Weng, 2010). In our environment, a firm cannot appropriate the benefits that its worker would obtain when switching from a LT to a ST contract because limited liability (which is a relevant constraint in the moral hazard problem) prevents decreasing the junior's salary. ${ }^{23}$

\footnotetext{
${ }^{23}$ The inefficiency does not depend on the level of the workers' outside option or on the property that type $L$ senior workers are not compensated beyond their outside option, as long as the limited liability constraint for the junior workers is binding.
} 
Next, we consider the case where $\widehat{R}$ is lower than $R^{o}$, that is, the population of firms has a high concentration of firms with a low $R$.

Theorem 2 Suppose $\widehat{R} \leq R^{o}$ and either $2 \widetilde{p}^{2}<p_{H}^{2}$ or $\widehat{R}$ is larger than a certain cut-off value $R_{a}<R^{o}{ }^{24}$ Then, if $p_{H}^{2}>\left(1+\frac{1}{\delta q}\right) p_{L}^{2}$ an equilibrium exists where

(i) firms with $R<\widehat{R}$ offer $S T$ contracts: $\widehat{w}_{J}^{S T}=\max \left\{(1+\delta) \underline{U}-\delta\left(q E_{H}+(1-q) E_{L}\right), 0\right\}$ to junior workers and $C_{L}^{S T}(R, \underline{U})$ to low-ability senior workers, (ii) firms with $R \geq \widehat{R}$ offer $S T$ contracts: $\widehat{w}_{J}^{S T}$ to junior workers and $C_{H}^{S T}\left(R, \widehat{U}_{H}\right)$ to highability senior workers, where $\widehat{U}_{H}$ is such that $E \widetilde{\pi}_{L}^{S T}\left(\widehat{R}, \widehat{w}_{J}^{S T}, \underline{U}\right)=E \widetilde{\pi}_{H}^{S T}\left(\widehat{R}, \widehat{w}_{J}^{S T}, \widehat{U}_{H}\right)$ (iii) junior workers accept both ST contracts with $\widehat{w}_{J}^{S T}$ and LT contracts that guarantee them $(1+\delta) \underline{U}$, and

(iv) senior workers accept contracts that guarantee them $\underline{U}$.

The basic trade-offs between the several contractual forms a firm can choose from do not depend on whether $\widehat{R}$ is higher or lower than $R^{o}$. Therefore, the intuition behind Theorem 2 is similar to that behind Theorem 1 . However, no firm is interested in offering LT contracts when $\widehat{R} \leq R^{o}$. ST contracts for low-ability agents provide higher profits than LT contracts for any firm with $R \leq R^{o}$ while ST contracts with high-ability agents are better than LT contracts for firms with $R \geq \widehat{R}$ (given $\widehat{w}_{J}^{S T}$ and $\widehat{U}_{H}$ ). Therefore, there is no space for the intermediate region in which LT contracts are the best alternative.

Theorem 2 presents a situation where, in equilibrium, the market is composed only of firms offering ST contracts. When $\widehat{R} \leq R^{o}$, most firms give a low value to success, as most firms are below $R^{o}$. Therefore, they care more about decreasing the cost of junior agents than about the additional incentives provided by LT contracts. In fact, firms with low $R$ may benefit from the existence of firms with high $R$ in such a way that even the marginal firm $\widehat{R}$ may strictly prefer ST contracts (either with low- or high-ability workers) than LT contracts! This occurs when $E_{H}>\widehat{U}_{H}$ (and $\widehat{w}_{J}^{S T}>0$ ), in which case junior workers accept very low salaries because they may migrate, as seniors, to firms with very high $R$, giving them high rents.

Finally, we consider the case where $\underline{R}$ is large (or $R^{o}$ is very small), that is, where only firms with high-valued projects are in the market.

\footnotetext{
${ }^{24}$ The cut-off value $R_{a}$ may be lower than $\underline{R}$, so that the theorem applies to all $\widehat{R} \leq R^{o}$. However, if $\underline{R}$ is very low and $2 \widetilde{p}^{2} \geq p_{H}^{2}$, then an equilibrium like the one described in the theorem may not exist if most of the firms have a very low productivity parameter $R$.
} 
Theorem 3 Suppose $R^{o}<\underline{R}$. Then, no equilibrium exists in which ST contracts are adopted.

When $\underline{R}>R^{o}$, all firms value their projects enough so that none is ready to offer ST contracts to permanently retain low-ability workers. They would rather offer LT agreements, which ensures them high-ability workers with some probability.

Finally, we discuss the extension of theorems 1 and 2 if the assumption $p_{H}^{2}>\left(1+\frac{1}{\delta q}\right) p_{L}^{2}$ does not hold. In what follows, we propose a constructive way to find an equilibrium for any set of parameters. As above, we denote by $R^{o}$ the unique value that satisfies $E \widetilde{\pi}^{L T}\left(R^{o}\right)=E \widetilde{\pi}_{L}^{S T}\left(R^{o}, 0, \underline{U}\right)$. In the case where $R^{o}<\underline{R}$, we are in the framework of Theorem 3. Therefore, we analyse the cases where $R^{o} \geq \underline{R}$.

Consider first a market where $R^{o}<\widehat{R}$. We can then proceed in a similar manner as in Theorem 1 . We denote by $R^{o o}$ a firm such that $q G\left(R^{o}\right)=(1-q) G\left(R^{o o}\right)$ and by $U_{H}^{o o}$ the level of utility that makes the firm $R^{o o}$ indifferent between using LT contracts and hiring high-ability senior workers through ST contracts when the junior salary is $w_{J}^{S T}=0$, that is, $E \widetilde{\pi}^{L T}\left(R^{o o}\right)=E \widetilde{\pi}_{H}^{S T}\left(R^{o o}, 0, U_{H}^{o o}\right)$. If the agents' participation constraint is satisfied for $U_{H}^{o o}$, that is, $\delta q U_{H}^{o o}+\delta(1-q) \underline{U} \geq \underline{U}+\delta \underline{U}$, then Theorem 1 holds. (For example, under assumption $p_{H}^{2}>\left(1+\frac{1}{\delta q}\right) p_{L}^{2}$, the participation constraint always holds.) For the remainder of the discussion, it is useful to briefly comment on the method of proof in Theorem 1: We begin by computing the value $U_{H}^{o}$ such that $E \widetilde{\pi}^{L T}\left(R^{o}\right)=E \widetilde{\pi}_{H}^{S T}\left(R^{o}, 0, U_{H}^{o}\right)$. We then show that $\delta q U_{H}^{o}+\delta(1-q) \underline{U}=\underline{U}+\delta \underline{U}$. Then, given that $U_{H}^{o o}>U_{H}^{o}$ because $R^{o o}>R^{o}$, we observe that the participation constraint is also satisfied for $U_{H}^{o o}$. If $p_{H}^{2} \leq\left(1+\frac{1}{\delta q}\right) p_{L}^{2}$, we can also compute $R^{o}$ and $U_{H}^{o} \cdot{ }^{25}$ However, we can not immediately extend the previous argument because, in this case, $\delta q U_{H}^{o}+\delta(1-q) \underline{U}<\underline{U}+\delta \underline{U}$. Therefore, Theorem 1 only holds with the proposed contracts if $U_{H}^{o o}$ is sufficiently high compared to $U_{H}^{o}$, that is, when $R^{o o}$ is high enough. ${ }^{26}$

In the case where $R^{o}<\widehat{R}$ but $\delta q U_{H}^{o o}+\delta(1-q) \underline{U}<\underline{U}+\delta \underline{U}$, we adapt the method to find an equilibrium as follows. We define $R^{o}(w), R^{o o}(w)$, and $U_{H}^{o o}(w)$ as before, except

\footnotetext{
${ }^{25}$ In particular, $R^{o}=\frac{1}{\delta\left[\tilde{p} \sqrt{1+\frac{1}{\delta}}-p_{L}\right]} \sqrt{2 \underline{U}}$ if $p_{H}^{2} \in\left[\left(1+\frac{1}{4 \delta(1+\delta) q}\right) p_{L}^{2},\left(1+\frac{1}{\delta q}\right) p_{L}^{2}\right]$ and $R^{o}=$

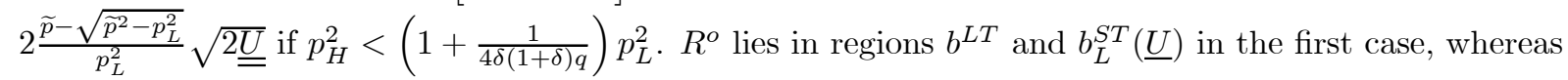
$R^{o}$ lies in regions $b^{L T}$ and $c_{L}^{S T}(\underline{U})$ in the second case.

${ }^{26}$ Note that we can apply Lemma 1 (c) if $U_{H}^{o o}$ satisfies the agent's participation constraint and $w_{J}^{S T}=0$ because, in this case, $U_{H}^{o o}$ is always higher than $\frac{\widetilde{p}^{2}}{p_{H}^{2}} \underline{\underline{U}}$.
} 
that the values are computed when the junior salary is $w_{J}^{S T}=w>0 .{ }^{27}$ We look for the salary $w^{*}$ such that $w^{*}+\delta q U_{H}^{o o}\left(w^{*}\right)+\delta(1-q) \underline{U}=\underline{U}+\delta \underline{U}$. If $R^{o}\left(w^{*}\right) \geq \underline{R}$, then an equilibrium similar to the one presented in Theorem 1 holds. In this case, the regions and equilibrium contracts are obtained by substituting $R^{o}\left(w^{*}\right), R^{o o}\left(w^{*}\right)$, and $U_{H}^{o o}\left(w^{*}\right)$ for $R^{o}, R^{o o}$, and $U_{H}^{o o}$ in Theorem $1 .{ }^{28}$ On the other hand, if $R^{o}\left(w^{*}\right)<\underline{R}$, then there is no equilibrium where firms offer ST contracts, and Theorem 3 applies.

Consider now a market where $R^{o} \geq \widehat{R}$. We then denote by $w^{\prime}$ the salary such that $R^{o}\left(w^{\prime}\right)=\widehat{R}$. We distinguish two cases. If the participation constraint does not hold for $w^{\prime}$, that is, if $w^{\prime}+\delta q U_{H}^{o o}\left(w^{\prime}\right)+\delta(1-q) \underline{U}<\underline{U}+\delta \underline{U}$, then we proceed as in the previous paragraphs until we find $w^{*}$. Therefore, either an equilibrium similar to Theorem 1 (for $\left.w^{*}\right)$ or Theorem 3 holds, depending on whether $R^{o}\left(w^{*}\right)$ is lower or higher than $\underline{R}$. On the other hand, if $w^{\prime}+\delta q U_{H}^{o o}\left(w^{\prime}\right)+\delta(1-q) \underline{U} \geq \underline{U}+\delta \underline{U}$, then the equilibrium proposed in Theorem 2, where only ST contracts are signed, holds.

\section{Endogenous set of firms}

In this section, we briefly comment on the hypothesis concerning the set of active firms. We have assumed that the set of firms active in the market is fixed and measured by the distribution function $G(R)$ on the set $[\underline{R}, \bar{R}]$. Therefore, we have made the implicit assumption that, irrespective of the market outcome, all firms find it worthwhile to remain active in the market. This assumption can be sustained either because the worst firm has a sufficiently profitable project (i.e., $\underline{R}$ is high enough) or because the fixed component of the firms' activity $(F)$ is sufficiently high. However, in general, the set of active firms is endogenous; it depends on the profitability of the market.

Suppose that the set of potential firms is distributed in the interval $[0, \bar{R}]$, according to some distribution function $J(R)$. The set of active firms $[\underline{R}, \bar{R}]$ will be determined by the condition that, in equilibrium, the profits of firm $\underline{R}$ are zero and the distribution $G(R)$ is derived from $J(R)$. The level of $\underline{R}$ will depend on the values of the parameters in the

\footnotetext{
${ }^{27} R^{o}(w)<\widehat{R}$ for all $w$ because $R^{o}(w)$ is decreasing in $w$. Moreover, $R^{o o}(w)$ is decreasing in $w$.

${ }^{28}$ The salary $w^{*}$ always exists and is not larger than $\underline{U}$ because $U_{H}^{o o}(w)>\underline{U}$ for all $w$. We also note that, as in Theorem 2, the proposed equilibrium might not hold if $2 \widetilde{p}^{2} \geq p_{H}^{2}$ or, after the process, $U_{H}^{o o}$ is lower than $\frac{\widetilde{p}^{2}}{p_{H}^{2}} \underline{\underline{U}}$ so that we cannot apply Lemma 1 (c). The same caveat applies to the candidate proposed in the next paragraph.
} 
model. We can discuss, for example, how changes in the parameter $F$ affect the set of active firms and the type of contracts signed in the market.

If $F$ is very low, then the minimum $\underline{R}$ (and also $\widehat{R}$ ) is high. Therefore, $R^{o}$ (which does not depend on the distribution of firms) is lower than $\underline{R}$ and, in equilibrium, only LT contracts are signed. When $F$ increases, both $\underline{R}$ and $\widehat{R}$ decrease until we obtain the region where ST contracts appear for low and high values of $R$. Additional increases in $F$ cause the regions in which there are ST contracts to expand until only ST contracts exist in equilibrium. Therefore, we should expect a prevalence of ST contracts in those markets where the "fixed" component of revenue (that is, the component that does not depend on incentives) is large, whereas we should observe LT contracts in those markets in which most income is earned through work that is subject to moral hazard. This, in fact, reflects the main trade-off that firms face. Focusing solely on incentives, LT contracts are better as they can exploit the intertemporal nature of the firm/worker relationship to alleviate the moral hazard problem. On the other hand, ST contracts offer an added flexibility that improves the efficiency of firm/worker matching. If market characteristics are such that incentives play an important role in all firms' profits, then LT contracts prevail. However, if appropriate worker selection is the key issue, then ST contracts are used in equilibrium.

\section{Conclusion}

We have introduced and analysed an equilibrium model to discuss the advantages and disadvantages of short-term versus long-term contracts in a dynamic environment where senior workers are subject to moral hazard. On the one hand, long-term contracts are better for providing incentives because they enable firms to credibly transfer payments from earlier to later periods over the term of a worker's employment, and this transfer alleviates the incentive compatibility constraint. On the other hand, short-term contracts allow the market to achieve better matching between the abilities of agents and the needs of firms. Those agents who turn out to have high ability can be hired by firms that profit the most from employing them.

When firms operating very profitable projects ${ }^{29}$ identify talented workers, they are

\footnotetext{
${ }^{29}$ Projects for which the incentives of the senior workers play an important role in firms' profits (firms with high $R$ ).
} 
willing to offer them high salaries or bonuses. High-ability seniors end up receiving high remuneration when short-term contracts are in place, which in turn results in reduced pay for juniors, who foresee the prospects of high wages as seniors. Consequently, firms with less lucrative ventures ${ }^{30}$ may not be able to retain high-ability workers, but they indirectly profit from the existence of these workers because they allow the firms to hire junior workers at a much lower cost.

We find equilibria in which two types of firms use short-term contracts: firms for which the success of a project strongly depends on the senior worker's effort and that generally end up hiring high-ability senior workers, and firms whose profits do not greatly depend on effort and that therefore hire low-ability senior workers. Intermediate firms may use long- or short-term contracts, depending on various market characteristics.

In addition to the equilibrium with short-term contracts, a situation that often exists, there always exists an equilibrium in which all firms choose long-term contracts (see Proposition 1). However, we argue that, in our modelling environment, whenever an equilibrium with short-term contracts and one with only long-term contracts coexist, the former is more "robust" as the latter is a "knife-edge" result. The outcome in which all firms use long-term contracts is sustained by the fact that, if no other firms offer shortterm contracts, no firm can profit from the enhanced flexibility that short-term contracts provide. However, a small subset of the best and worst firms has incentives to switch from long-term to short-term contracts. Given the equilibrium salary that would accrue to high-ability workers following this such a deviation, junior agents would be willing to accept low salaries and all the firms that deviate would obtain higher profits.

Finally, we comment on the assumption of symmetric information between firms and workers. A natural and interesting extension of our model, beyond the scope of this paper, is the introduction of private information about agents' abilities. If young workers have an informational advantage regarding over firms regarding their future managing ability, the choice of contract takes on an additional role as a screening mechanism. In this line, Matutes et al. (1994) present the choice of compensation schemes by two firms that compete in a labour market in which agents are heterogeneous and have private information about their skills. The authors show that, in equilibrium, one firm may offer a wage rate, whereas another may offer a piece rate. By proposing different compensation

\footnotetext{
${ }^{30}$ Firms in which the role played by the senior worker is less important to the outcome.
} 
schemes, firms induce self-selection among workers. Similarly, Loh (1994) discusses how employment probation can serve as a sorting device, as it induces self-selection by workers. Firms offering probationary employment tend to attract workers who are more confident in their capabilities.

In the framework of our paper, firms choose both the intensity of incentives and the length of contracts. If young workers have private information about their abilities, firms may try to screen them when they are young, which would induce a matching between heterogenous firms and heterogenous agents already in the first period of agents' lives. For example, assume young workers have perfect information about their type. Then, firms can compete for the high-ability workers through LT contracts that separate types. Firms with high $R$ can hire high-ability workers by offering them large second-period incentives while proposing extremely low payoffs for low-ability workers. Firms with low $R$ can hire low-ability workers through an optimal LT contract. In this case, in equilibrium, the efficient assignment is achieved from the very beginning and we only observe LT contracts in equilibrium: they allow for screening of workers and a better provision of incentives. The advantage of ST contracts as a sorting mechanism vanishes.

However, if young workers have private but incomplete information about their abilities, then ST contracts may still play a role. Consider a situation where young agents have more precise information than firms have about the probability that they will turn out to be high-ability senior workers; agents' types will become public information once they have worked as junior workers. The previous argument then suggests that the region where LT contracts are optimal would expand, but the advantage of ST contracts as a sorting device remains.

\section{Appendix}

Proof of Proposition 1. We first note that, in a situation in which all firms sign LT contracts, if a firm follows the strategy of offering ST contracts to its workers, it necessarily hires as senior agent at period $t$ the same agent that it hired as a junior at period $t-1$. Also, the only alternative occupation for the senior agent is to get out of the market, since no other firm is interested in hiring him, independent on his ability. Then, any sequence of ST contracts can be replicated as a LT contract. Therefore, the optimal ST contracts cannot provide higher profits than the optimal LT contracts.

Proof of Proposition 2. Substituting $e_{H}$ and $e_{L}$ by their value and multiplying 
the objective function by $\delta$, the firm's program can be rewritten as:

$$
\begin{gathered}
\max _{\left(w_{J}, w_{H}, \Delta_{H}, w_{L}, \Delta_{L}\right)} \delta F+\delta q\left(\frac{1}{2} p_{H}^{2} \Delta_{H}\left(R-\Delta_{H}\right)-w_{H}\right)+\delta(1-q)\left(\frac{1}{2} p_{L}^{2} \Delta_{L}\left(R-\Delta_{L}\right)-w_{L}\right)-w_{J} \\
\text { s.t. } w_{J}+\delta q\left(\frac{1}{2} p_{H}^{2} \Delta_{H}^{2}+w_{H}\right)+\delta(1-q)\left(\frac{1}{2} p_{L}^{2} \Delta_{L}^{2}+w_{L}\right) \geq(1+\delta) \underline{U} \\
w_{J} \geq 0, \quad w_{H} \geq 0, \quad w_{L} \geq 0 .
\end{gathered}
$$

Let $\lambda, \mu, \rho_{H}$ and $\rho_{L}$ be the Lagrange multipliers corresponding to the constraints. The Kuhn-Tucker (first-order) conditions of the above maximization problem include the constraints, and the non-negativity of the multipliers: $\lambda \geq 0, \mu \geq 0, \rho_{H} \geq 0, \rho_{L} \geq 0$. The derivatives of the Lagrangian with respect to $\Delta_{H}$ and $\Delta_{L}$ are:

$$
\begin{gathered}
\delta q p_{H}^{2}\left(R-2 \Delta_{H}\right)+\lambda \delta q p_{H}^{2} \Delta_{H}=0 \\
\delta(1-q) p_{L}^{2}\left(R-2 \Delta_{L}\right)+\lambda \delta(1-q) p_{L}^{2} \Delta_{L}=0 .
\end{gathered}
$$

Equations (2) and (3) imply that $\Delta_{H}=\Delta_{L}$, which we denote $\Delta$ in the rest of the proof. The derivatives of the Lagrangian with respect to $w_{J}, w_{H}$ and $w_{L}$ are:

$$
\begin{aligned}
-1+\lambda+\mu & =0 \\
-\delta q+\lambda \delta q+\rho_{H} & =0 \\
-\delta(1-q)+\lambda \delta(1-q)+\rho_{L} & =0,
\end{aligned}
$$

which imply $\mu=1-\lambda, \rho_{H}=\delta q(1-\lambda)$ and $\rho_{L}=\delta(1-q)(1-\lambda)$; therefore, either the three constraints are binding or none is. The last Kuhn-Tucker conditions are:

$$
\begin{gathered}
\lambda\left[w_{J}+\delta\left[q\left(w_{H}+\frac{1}{2} p_{H}^{2} \Delta_{H}^{2}\right)+(1-q)\left(w_{L}+\frac{1}{2} p_{L}^{2} \Delta_{L}^{2}\right)\right]-(1+\delta) \underline{U}\right]=0 \\
\mu w_{J}=0, \rho_{H} w_{H}=0 \text { and } \rho_{L} w_{L}=0 .
\end{gathered}
$$

From (2) and (4) we can deduce that:

$$
\lambda=2-\frac{R}{\Delta} \quad \text { and } \quad \rho_{H}=\delta q\left(\frac{R}{\Delta}-1\right)
$$

We study the different regions where the Kuhn-Tucker conditions may be satisfied:

Region $a^{L T}: \mu=\rho_{H}=\rho_{L}=0$. Then $\lambda=1$ and $\Delta=R$. We write the participation constraint as

$$
w_{J}+\delta\left(q w_{H}+(1-q) w_{L}\right)+\delta \frac{1}{2} R^{2} \widetilde{p}^{2}=(1+\delta) \underline{U} .
$$


Any combination of $w_{J}, w_{H}$ and $w_{L}$ that satisfies the previous constraint and such that the three values are larger or equal to 0 constitutes an optimal solution (in particular, the values proposed in the proposition). This type of solution can exist only if $w_{J}+$ $\delta\left(q w_{H}+(1-q) w_{L}\right) \geq 0$, that is $R \leq \frac{1}{\widetilde{p}} \sqrt{2 \underline{\underline{U}}}$.

Region $b^{L T}: \mu>0, \rho_{H}>0, \rho_{L}>0, \lambda>0$. Payments when young and in case of failure are $w_{J}=w_{H}=w_{L}=0$ and the bonus in case of success is $\Delta=\frac{1}{\widetilde{p}} \sqrt{2 \underline{\underline{U}}}$. Finally, this is a candidate only if $\lambda \in[0,1]$, i.e., $R \in\left[\frac{1}{\widetilde{p}} \sqrt{2 \underline{\underline{U}}}, \frac{2}{\widetilde{p}} \sqrt{2 \underline{\underline{U}}}\right]$. The candidate at the lower bound of this region coincides with the candidate at the higher bound of Region $a^{L T}$.

Region $c^{L T}: \mu>0, \rho_{H}>0, \rho_{L}>0, \lambda=0$. Then $w_{J}=w_{H}=w_{L}=0$, and $\Delta=\frac{R}{2}$. In this case the participation constraint holds only if $R \geq \frac{2}{\widetilde{p}} \sqrt{2 \underline{\underline{U}}}$. The candidate at the lower bound of this region coincides with the candidate at the higher bound of Region $b^{L T}$.

The unique candidate for each value of $R$ is the optimal solution of the firm's maximization program. From the optimal contract in each case, it is immediate to compute agent's effort(s) and utility, and firm's profits. Additionally, easy calculations show that the function $E \widetilde{\pi}^{L T}(R)$ is continuously differentiable in $R$.

Proof of Proposition 3. Substituting $e_{i}$ by its value in the firm's program, we can rewrite it as

$$
\begin{array}{cc}
\max _{\left(w_{i}, \Delta_{i}\right)} F+ & p_{i}^{2} \Delta_{i}\left(R-\Delta_{i}\right)-w_{i} \\
\text { s.t. } & \frac{1}{2} p_{i}^{2} \Delta_{i}^{2}+w_{i} \geq U_{i} \\
& w_{i} \geq 0 .
\end{array}
$$

Let $\lambda, \rho$ be the Lagrange multipliers corresponding to the constraints. The Kuhn-Tucker (first-order) conditions of the above maximization problem include the constraints, and the non-negativity of the multipliers: $\lambda \geq 0, \rho \geq 0$. The derivatives of the Lagrangian with respect to $w_{i}$ and $\Delta_{i}$ are

$$
\begin{gathered}
-1+\lambda+\rho=0, \\
p_{i}^{2}\left(R-2 \Delta_{i}\right)+\lambda p_{i}^{2} \Delta_{i}=0 .
\end{gathered}
$$

From (5) and (6) we can deduce that:

$$
\lambda=2-\frac{R}{\Delta_{i}} \quad \text { and } \quad \rho=\frac{R}{\Delta_{i}}-1 .
$$

We study the different regions.

Region $a_{i}^{S T}\left(U_{i}\right): \rho=0$. Then $\Delta_{i}=R$, which implies $\lambda=1>0$. The participation 
constraint is $\frac{1}{2} p_{i}^{2} R^{2}+w_{i}=U_{i}$. Therefore, $w_{i}=U_{i}-\frac{1}{2} p_{i}^{2} R^{2} \geq 0$ if and only if $R \leq \frac{1}{p_{i}} \sqrt{2 U_{i}}$. Region $b_{i}^{S T}\left(U_{i}\right): \rho>0, \lambda>0$. Payments are $w_{i}=0$ and $\Delta_{i}=\frac{1}{p_{i}} \sqrt{2 U_{i}}$. This is a candidate only if $\lambda \geq 0$ and $\rho \geq 0$, i.e., $R \in\left[\frac{1}{p_{i}} \sqrt{U_{i}}, \frac{2}{p_{i}} \sqrt{2 U_{i}}\right]$.

Region $c_{i}^{S T}\left(U_{i}\right): \rho>0, \lambda=0$. Then $w_{i}=0$, and $\Delta_{i}=\frac{R}{2}$. In this case the participation constraint holds only if $R \geq \frac{2}{p_{i}} \sqrt{2 U_{i}}$.

The unique candidate for each value of $R$ is the optimal solution of the firm's maximization program. From the optimal contract in each case, it is immediate to compute agent's effort(s) and utility.

Proof of Lemma 1. We highlight that the three derivatives that we consider in the lemma, $\frac{\partial E \widetilde{\pi}_{L}^{S T}}{\partial R}\left(R, w_{J}, \underline{U}\right), \frac{\partial E \widetilde{\pi}^{L T}}{\partial R}(R)$, and $\frac{\partial E \widetilde{\pi}_{H}^{S T}}{\partial R}\left(R, w_{J}, U_{H}\right)$, have a similar shape: they are first linear in $R$ until they reach some $R_{1}$ (either $\frac{1}{p_{L}} \sqrt{2 \underline{U}}$, or $\frac{1}{\widetilde{p}} \sqrt{2 \underline{\underline{U}}}$, or $\frac{1}{p_{H}} \sqrt{2 U_{H}}$ ), then they are constant until they reach a second threshold $R_{2}$ and, from $R_{2}$ on, they are linear in $R$ again. The proof of the three parts in the lemma is similar. We write a complete proof of part (a) and we point out the main elements of parts (b) and (c).

(a) First, notice that if $R$ lies in both regions $a_{L}^{S T}(\underline{U})$ and $a^{L T}, \frac{\partial E \widetilde{\pi}_{L}^{S T}}{\partial R}=p_{L}^{2} R<\widetilde{p}^{2} R=$ $\frac{\partial E \tilde{\pi}^{L T}}{\partial R}$. The same comparison holds if $R$ lies in both regions $c_{L}^{S T}(\underline{U})$ and $c^{L T}$. Additionally, if $R$ lies in both regions $b_{L}^{S T}(\underline{U})$ and $b^{L T}, \frac{\partial E \widetilde{\pi}_{L}^{S T}}{\partial R}=p_{L} \sqrt{2 \underline{U}}<\widetilde{p} \sqrt{2 \underline{\underline{U}}}=\frac{\partial E \widetilde{\pi}^{L T}}{\partial R}$.

Second, if $\frac{1}{\tilde{p}} \sqrt{2 \underline{\underline{U}}} \geq \frac{1}{p_{L}} \sqrt{2 \underline{U}}$ (and $\frac{2}{\widetilde{p}} \sqrt{2 \underline{\underline{U}}} \geq \frac{2}{p_{L}} \sqrt{2 \underline{U}}$ ), then $\frac{\partial E \widetilde{\pi}^{L T}}{\partial R}$ is increasing in a larger region of parameters than $\frac{\partial E \tilde{\pi}_{L}^{S T}}{\partial R}$ before becoming constant (at a higher level than $\frac{\partial E \tilde{\pi}_{L}^{S T}}{\partial R}$ in region $\left.b_{L}^{S T}(\underline{U})\right)$. Finally, even if $\frac{\partial E \tilde{\pi}_{L}^{S T}}{\partial R}$ starts increasing again (i.e., it reaches region $c_{L}^{S T}(\underline{U})$ ) before $\frac{\partial E \tilde{\pi}^{L T}}{\partial R}$ (because $\frac{2}{p_{L}} \sqrt{2 \underline{U}} \leq \frac{2}{\widetilde{p}} \sqrt{2 \underline{\underline{U}}}$ ), it is always lower than the latter because it is lower even when $R=\frac{2}{\widetilde{p}} \sqrt{2 \underline{\underline{U}}}$, given that we have seen that $\frac{\partial E \widetilde{\pi}_{L}^{S T}}{\partial R}<\frac{\partial E \widetilde{\pi}^{L T}}{\partial R}$ for any $R$ which lies in both regions $c_{L}^{S T}(\underline{U})$ and $c^{L T}$.

Third, suppose $\frac{1}{\widetilde{p}} \sqrt{2 \underline{\underline{U}}}<\frac{1}{p_{L}} \sqrt{2 \underline{U}}$ (and $\frac{2}{\widetilde{p}} \sqrt{2 \underline{\underline{U}}}<\frac{2}{p_{L}} \sqrt{2 \underline{U}}$ ). Given that $\frac{\partial E \tilde{\pi}_{L}^{S T}}{\partial R}$ is smaller than $\frac{\partial E \widetilde{\pi}^{L T}}{\partial R}$ when $\frac{\partial E \widetilde{\pi}_{L}^{S T}}{\partial R}$ reaches the region where it becomes constant, and that it is certainly also smaller when it starts increasing again (because $\frac{\partial E \pi^{L T}}{\partial R}$ has reached this region before), it is not possible that the two derivatives cross. Therefore, $\frac{\partial E \widetilde{\pi}_{L}^{S T}}{\partial R}<\frac{\partial E \tilde{\pi}^{L T}}{\partial R}$ for any $R>0$.

(b) If $R$ lies in both regions $a_{L}^{S T}(\underline{U})$ and $a_{H}^{S T}\left(U_{H}\right), \frac{\partial E \tilde{\pi}_{L}^{S T}}{\partial R}=p_{L}^{2} R<p_{H}^{2} R=\frac{\partial E \tilde{\pi}_{H}^{S T}}{\partial R}$. The same comparison holds in regions $c_{L}^{S T}(\underline{U})$ and $c_{H}^{S T}\left(U_{H}\right)$. Also, if $R$ lies in both regions $b_{L}^{S T}(\underline{U})$ and $b_{H}^{S T}\left(U_{H}\right), \frac{\partial E \widetilde{\pi}_{L}^{S T}}{\partial R}=p_{L} \sqrt{2 \underline{U}}<p_{H} \sqrt{2 U_{H}}=\frac{\partial E \widetilde{\pi}_{H}^{S T}}{\partial R}$. The rest of the proof is identical to the one in part (a). 
(c) For $R$ in both regions $a^{L T}$ and $a_{H}^{S T}\left(U_{H}\right)$ (and similarly in $c^{L T}$ and $c_{H}^{S T}\left(U_{H}\right)$ ), $\frac{\partial E \widetilde{\pi}^{L T}}{\partial R}=\widetilde{p}^{2} R<p_{H}^{2} R=\frac{\partial E \widetilde{\pi}_{H}^{S T}}{\partial R}$. If $R$ lies in both regions $b^{L T}$ and $b_{H}^{S T}\left(U_{H}\right)$, then $\frac{\partial E \widetilde{\pi}^{L T}}{\partial R}=$ $\widetilde{p} \sqrt{2 \underline{\underline{U}}}<p_{H} \sqrt{2 U_{H}}=\frac{\partial E \tilde{\pi}_{H}^{S T}}{\partial R}$ if and only if $U_{H}>\frac{\widetilde{p}^{2}}{p_{H}^{2}} \underline{\underline{U}}$. If this inequality holds, the rest of the proof of part (c) is identical to the one in part (a). Otherwise, suppose $U_{H} \leq \frac{\widetilde{p}^{2}}{p_{H}^{2}} \underline{U}$ and $2 \widetilde{p}^{2}<p_{H}^{2}$. In this case, $\frac{2}{p_{H}} \sqrt{2 U_{H}}<\frac{1}{\widetilde{p}} \sqrt{2 \underline{\underline{U}}}$ (because $U_{H} \leq \frac{\widetilde{p}^{2}}{p_{H}^{2}} \underline{\underline{U}}<\frac{1}{2} \underline{\underline{U}}<\frac{1}{4} \underline{\underline{U}}$ ). Notice that $\frac{\partial E \widetilde{\pi}^{L T}}{\partial R}<\frac{\partial E \tilde{\pi}_{H}^{S T}}{\partial R}$ up to $\frac{1}{p_{H}} \sqrt{2 U_{H}}, \frac{\partial E \tilde{\pi}_{H}^{S T}}{\partial R}$ is constant in $\left[\frac{1}{p_{H}} \sqrt{2 U_{H}}, \frac{2}{p_{H}} \sqrt{2 U_{H}}\right]$, $\frac{\partial E \widetilde{\pi}^{L T}}{\partial R}$ is constant in $\left[\frac{1}{\widetilde{p}} \sqrt{2 \underline{\underline{U}}}, \frac{2}{\widetilde{p}} \sqrt{2 \underline{\underline{U}}}\right]$ and $\frac{\partial E \widetilde{\pi}^{L T}}{\partial R}<\frac{\partial E \widetilde{\pi}_{H}^{S T}}{\partial R}$ from $\frac{2}{\widetilde{p}} \sqrt{2 \underline{\underline{U}}}$ (therefore, also from $\frac{1}{\widetilde{p}} \sqrt{2 \underline{\underline{U}}}$ on). Therefore, if $\frac{\partial E \tilde{\pi}^{L T}}{\partial R} \geq \frac{\partial E \tilde{\pi}_{H}^{S T}}{\partial R}$ at some $R$, it is necessarily the case that the inequality holds either at $R=\frac{2}{p_{H}} \sqrt{2 U_{H}}$ or $R=\frac{1}{\widetilde{p}} \sqrt{2 \underline{\underline{U}}}$. Notice that both $R=$ $\frac{2}{p_{H}} \sqrt{2 U_{H}}$ and $\frac{1}{\widetilde{p}} \sqrt{2 \underline{\underline{U}}}$ lye in regions $a^{L T}$ and $\left.c_{H}^{S T}\left(U_{H}\right)\right)$. However, $\frac{\partial E \tilde{\pi}^{L T}}{\partial R}\left(R=\frac{2}{p_{H}} \sqrt{2 U_{H}}\right)=$ $\widetilde{p}^{2} \frac{2}{p_{H}} \sqrt{2 U_{H}}<\frac{1}{2} p_{H}^{2} \frac{2}{p_{H}} \sqrt{2 U_{H}}=\frac{\partial E \widetilde{\pi}_{H}^{S T}}{\partial R}\left(R=\frac{2}{p_{H}} \sqrt{2 U_{H}}\right)$ because $\widetilde{p}^{2}<\frac{1}{2} p_{H}^{2}$ and, for the same reason, $\frac{\partial E \tilde{\pi}^{L T}}{\partial R}\left(R=\frac{1}{\tilde{p}} \sqrt{2 \underline{\underline{U}}}\right)<\frac{\partial E \tilde{\pi}_{H}^{S T}}{\partial R}\left(R=\frac{1}{\tilde{p}} \sqrt{2 \underline{\underline{U}}}\right)$.

Proof of Theorem 1. We do the proof through a series of claims.

Claim 1: If $w_{J}^{S T}=0$, then $R^{o}<\frac{1}{p_{L}} \sqrt{2 \underline{U}}$ and $R^{o}<\frac{1}{\tilde{p}} \sqrt{2 \underline{\underline{U}}}$.

Proof of Claim 1: If the value $R^{o}$ that satisfies $E \widetilde{\pi}^{L T}\left(R^{o}\right)=E \widetilde{\pi}_{L}^{S T}\left(R^{o}, 0, \underline{U}\right)$ lies in both regions $a^{L T}$ and $a_{L}^{S T}(\underline{U})$ (i.e., $R^{o}<\frac{1}{\widetilde{p}} \sqrt{2 \underline{\underline{U}}}$ and $R^{o}<\frac{1}{p_{L}} \sqrt{2 \underline{U}}$ ), then from the expressions of $E \widetilde{\pi}^{L T}\left(R^{o}\right)$ and $E \widetilde{\pi}_{L}^{S T}\left(R^{o}, 0, \underline{U}\right)$ it follows that $R^{o}=\frac{1}{\sqrt{\delta q\left(p_{H}^{2}-p_{L}^{2}\right)}} \sqrt{2 \underline{U}}$. Moreover, it is easy to check that each of the inequalities $R^{o}<\frac{1}{p_{L}} \sqrt{2 \underline{U}}$ and $R^{o}<\frac{1}{\widetilde{p}} \sqrt{2 \underline{U}}$ is equivalent to $p_{H}^{2}>\left(1+\frac{1}{\delta q}\right) p_{L}^{2}$, which is the condition required in the theorem.

Claim 2: Consider the value $U_{H}^{o}$ such that $E \widetilde{\pi}^{L T}\left(R^{o}\right)=E \widetilde{\pi}_{H}^{S T}\left(R^{o}, 0, U_{H}^{o}\right)$ (that is, $\left.E \widetilde{\pi}_{L}^{S T}\left(R^{o}, 0, \underline{U}\right)=E \widetilde{\pi}_{H}^{S T}\left(R^{o}, 0, U_{H}^{o}\right)\right)$. If junior workers anticipate that they will obtain at least $U_{H}^{o}$ when senior if they turn out to be high-ability, then they are ready to accept $w_{J}^{S T}=0$.

Proof of Claim 2: We proceed as follows. We conjecture that $U_{H}^{o}$ is such that $R^{o}<$ $\frac{1}{p_{H}} \sqrt{2 U_{H}^{o}}$, we compute the corresponding $U_{H}^{o}$ in this region, and then we will show that it is indeed the case that $R^{o}<\frac{1}{p_{H}} \sqrt{2 U_{H}^{o}}$. Therefore, $U_{H}^{o}$ is defined by

$$
F-\underline{U}+\frac{1}{2} p_{L}^{2} R^{o 2}=F-U_{H}^{o}+\frac{1}{2} p_{H}^{2} R^{o 2}
$$

i.e., $U_{H}^{o}=\underline{U}+\frac{1}{2}\left(p_{H}^{2}-p_{L}^{2}\right) R^{o 2}$ or $U_{H}^{o}=\left(1+\frac{1}{\delta q}\right) \underline{U}$. For this value, $R^{o}<\frac{2 k}{p_{H}} \sqrt{U_{H}^{o}}$ if and only if $\frac{1}{\sqrt{\delta q\left(p_{H}^{2}-p_{L}^{2}\right)}}<\frac{1}{p_{H}} \sqrt{\left(1+\frac{1}{\delta q}\right)}$, i.e., $p_{L}^{2}<\delta q\left(p_{H}^{2}-p_{L}^{2}\right)$, which we have assumed. Finally, given $U_{H}^{o}$ and taking into account that $E_{H} \geq U_{H}^{o}$ and $E_{L} \geq \underline{U}$, a junior worker 
is ready to accept a ST contract with $w_{J}^{S T}$ whenever $w_{J}^{S T}+\delta q\left(1+\frac{1}{\delta q}\right) \underline{U}+\delta(1-q) \underline{U} \geq$ $(1+\delta) \underline{U}$, that is, whenever $w_{J}^{S T} \geq 0$.

Claim 3: $E \widetilde{\pi}_{L}^{S T}(R, 0, \underline{U}) \geq E \widetilde{\pi}_{H}^{S T}\left(R, 0, U_{H}\right)$ for any $U_{H} \geq U_{H}^{o}$ and for any $R \leq R^{o}$. Proof of Claim 3: Given the definition of $U_{H}^{o}$ in Claim 2, $E \widetilde{\pi}_{L}^{S T}\left(R^{o}, 0, \underline{U}\right) \geq E \widetilde{\pi}_{H}^{S T}\left(R^{o}, 0, U_{H}\right)$ for any $U_{H} \geq U_{H}^{o}$. Then, the claim follows after Lemma 1 (b).

Claim 4: $E \widetilde{\pi}_{L}^{S T}(R, 0, \underline{U}) \geq \max \left\{E \widetilde{\pi}_{H}^{S T}\left(R, 0, U_{H}^{o o}\right), E \widetilde{\pi}^{L T}(R)\right\}$ for any $R \leq R^{o}$. Proof of Claim 4: The first inequality follows after Claim 3, if we also take into account that $R^{o o}>R^{o}$ implies $U_{H}^{o o}>U_{H}^{o}$. The second inequality follows the definition of $R^{o}$ and Lemma 1 (a).

Claim 5: $E \widetilde{\pi}^{L T}(R) \geq \max \left\{E \widetilde{\pi}_{H}^{S T}\left(R, 0, U_{H}^{o o}\right), E \widetilde{\pi}_{L}^{S T}(R, 0, \underline{U})\right\}$ for any $R \in\left(R^{o}, R^{o o}\right)$. Proof of Claim 5: We first prove that we can apply Lemma 1 (c) because $U_{H}^{o o}>\frac{\widetilde{p}^{2}}{p_{H}^{2}} \underline{\underline{U}}$. Given that $U_{H}^{o o}>U_{H}^{o}$, the previous inequality holds if $\left(1+\frac{1}{\delta q}\right) \underline{U} \geq \frac{\widetilde{p}^{2}}{p_{H}^{2}} \frac{1}{\delta}(1+\delta) \underline{U}$, that is, $(\delta q+1) p_{H}^{2} \geq q(1+\delta) \widetilde{p}^{2}$, which is always satisfied. Then, the first part of the inequality follows after the characterisation of $U_{H}^{o o}$ in part (vi) of the theorem and Lemma 1 (c). The second part follows the definition of $R^{o}$ and Lemma 1 (a).

Claim 6: $E \widetilde{\pi}_{H}^{S T}\left(R, 0, U_{H}^{o o}\right) \geq \max \left\{E \widetilde{\pi}^{L T}(R), E \widetilde{\pi}_{L}^{S T}(R, 0, \underline{U})\right\}$ for any $R \geq R^{o o}$. Proof of Claim 6: By the same argument as in Claim 5, the maximum of the two terms inside the maximization is $E \widetilde{\pi}^{L T}(R)$. Then, the inequality is implied by the characterisation of $U_{H}^{o o}$ in part (vi) of the theorem, by the property that $U_{H}^{o o}>U_{H}^{o}$ and Lemma 1 (c).

Proof of Proposition 4. (i) In the discussion that precedes Proposition 4, we have shown that the equilibrium in Theorem 1 is preferred by the firms to that in Proposition 1. We now prove that this is also the case for the workers.

First, in Claim 2 in the proof of Theorem 1, we computed the value $U_{H}^{o}$ such that $E \widetilde{\pi}^{L T}\left(R^{o}\right)=E \widetilde{\pi}_{H}^{S T}\left(R^{o}, 0, U_{H}^{o}\right)$ and we showed that $\delta q U_{H}^{o}+\delta(1-q) \underline{U}=\underline{U}+\delta \underline{U}$, that is, $U_{H}^{o}=\left(1+\frac{1}{\delta q}\right) \underline{U}$. Given that $U_{H}^{o o}>U_{H}^{o}$ because $R^{o o}>R^{o}$, a sufficient condition for part (i) is that the workers are not worse-off in the equilibrium including ST contracts if we substitute $U_{H}^{o o}$ by $U_{H}^{o}$. Second, note that $\frac{2}{p_{H}} \sqrt{2 U_{H}^{o}} \leq \frac{2}{\widetilde{p}} \sqrt{2 \underline{\underline{U}}}$ because the inequality is equivalent to $\widetilde{p}^{2}\left(1+\frac{1}{\delta q}\right) \leq p_{H}^{2} \frac{1}{\delta}(1+\delta)$, that is, $\left(q p_{H}^{2}+(1-q) p_{L}^{2}\right)(\delta q+1) \leq q p_{H}^{2}(1+\delta)$, or, $p_{L}^{2} \leq\left(1+\frac{1}{\delta q}\right) p_{H}^{2} \delta q$. Third, the expected utility of a worker under a LT contract when $R>\frac{2}{\widetilde{p}} \sqrt{2 \underline{\underline{U}}}$ is (after easy computations) $U^{L T}(R)=\frac{1}{8} \delta \widetilde{p}^{2} R^{2}$ whereas the expected utility of a high-ability agent under a ST contract when $R>\frac{2}{p_{H}} \sqrt{2 U_{H}^{o}}$ is $U_{H}^{S T}(R)=\frac{1}{8} \delta p_{H}^{2} R^{2}$. 
It follows that the derivatives of the utilities satisfy $U_{H}^{S T \prime}(R)>U^{L T^{\prime}}(R)$, that is, the rent increases (with $R$ ) faster under ST contracts (with a high-ability worker) than under LT contracts. Fourth, from $\delta q U_{H}^{o}+\delta(1-q) \underline{U}=\underline{U}+\delta \underline{U}$ it follows that if there were no extra rents at any contract, then the total expected utility by workers would be the same in both equilibria. Given the second and third remarks, if there are extra rents, they involve a larger set of firms and they are higher for each $R$ under ST than under LT contracts. Therefore, the set of workers is also better-off in the equilibrium found in Theorem 1 than in that in Proposition 1.

(ii) By arguments similar to those before, shrinking the set of firms and workers that sign ST contracts in equilibrium cannot be beneficial: everyone involved would lose. On the other hand, a marginal expansion of the set of firms that use ST contracts in equilibrium improves efficiency. To show this property, consider that firms in the intervals $\left(R^{o}, R^{o}+\varepsilon\right]$ and $\left[R^{o o}-\mu, R^{o o}\right.$ ) (where $\varepsilon$ and $\mu$ are small and such that the proportion of firms in the intervals is the same as the rate of low- versus high-ability seniors) switch to ST contracts. Given that $E \widetilde{\pi}^{L T}(R)-E \widetilde{\pi}_{L}^{S T}(R, 0, \underline{U})=0$ for $R=R^{o}$, and the continuity of both profit functions in $R$, each firm in $\left(R^{o}, R^{o}+\varepsilon\right]$ looses an amount "approximately" equal to $\Delta\left(R^{o}\right)\left(R-R^{o}\right)$, where $\Delta\left(R^{o}\right) \equiv \frac{\partial\left(E \tilde{\pi}^{L T}(R)-E \widetilde{\pi}_{L}^{S T}(R, 0, \underline{U})\right)}{\partial R}$ and the derivative is evaluated at $R=R^{o}$. Also, the density of firms with a project of value $R$ is $g(R)$, where $g($.$) is the density function of G($.$) . Therefore, the total loss in profits for this set of$ firms is on the order of $\Delta\left(R^{o}\right) g\left(R^{o}\right) \varepsilon^{2}$. Similarly, the total loss in profits for the firms in $\left[R^{o o}-\mu, R^{o o}\right)$ is proportional to $g\left(R^{o o}\right) \mu^{2}$. Concerning the workers, the expected utility of a worker who is now hired under a ST contracts instead of a LT contract increases by a constant, following the arguments developed in part (i). Hence, the total utility gain by the workers in proportional to $\left(g\left(R^{o}\right) \varepsilon+g\left(R^{o o}\right) \mu\right)$. Given that we do the analysis for a fixed $U_{H}^{o o}$, those firms and workers are the only ones affected by the change. Therefore, for small $\varepsilon$ and $\mu$, the sum of profits and utility increases after the firms in the intervals $\left(R^{o}, R^{o}+\varepsilon\right]$ and $\left[R^{o o}-\mu, R^{o o}\right)$ switch from LT to ST contracts.

Proof of Theorem 2. Given that the behavior of the workers is optimal by construction, we prove the theorem if we show that firms' strategies are optimal. We do it through a series of claims.

Claim 1: $\widehat{w}_{J}^{S T} \leq \underline{U}-\frac{1}{2} \delta q \widehat{R}^{2}\left(p_{H}^{2}-p_{L}^{2}\right)$.

Proof of Claim 1. Given that $E_{H} \geq \widehat{U}_{H}$ and $E_{L} \geq \underline{U},(1+\delta) \underline{U}-\delta\left(q E_{H}+(1-q) E_{L}\right) \leq$ 
$\underline{U}-\frac{1}{2} \delta q \widehat{R}^{2}\left(p_{H}^{2}-p_{L}^{2}\right)$. Moreover $0 \leq \underline{U}-\frac{1}{2} \delta q \widehat{R}^{2}\left(p_{H}^{2}-p_{L}^{2}\right)$ because this inequality is equivalent to $\widehat{R} \leq \frac{1}{\sqrt{\delta q\left(p_{H}^{2}-p_{L}^{2}\right)}} \sqrt{2 \underline{U}}=R^{o}$.

Claim 2: $\widehat{R} \leq \frac{1}{p_{H}} \sqrt{2 \widehat{U}_{H}}$ and $\widehat{R} \leq \frac{1}{p_{L}} \sqrt{2 \underline{U}}$.

Proof of Claim 2: We assume that $\widehat{U}_{H}$ such that $E \widetilde{\pi}_{L}^{S T}\left(\widehat{R}, \widehat{w}_{J}^{S T}, \underline{U}\right)=E \widetilde{\pi}_{H}^{S T}\left(\widehat{R}, \widehat{w}_{J}^{S T}, \widehat{U}_{H}\right)$ satisfies that $\widehat{R}$ lies in regions $a_{L}^{S T}(\underline{U})$ and $a_{H}^{S T}\left(\widehat{U}_{H}\right)$ and we will prove that this is indeed the case. If $\widehat{R}$ lies in these regions, then $\widehat{U}_{H}=\underline{U}+\frac{1}{2} \widehat{R}^{2}\left(p_{H}^{2}-p_{L}^{2}\right)$. Therefore, $\widehat{R} \leq \frac{1}{p_{H}} \sqrt{2 \widehat{U}_{H}}=\frac{1}{p_{H}} \sqrt{2 \underline{U}+\widehat{R}^{2}\left(p_{H}^{2}-p_{L}^{2}\right)}$ if and only if $\widehat{R} \leq \frac{1}{p_{L}} \sqrt{2 \underline{U}}$, which is implied by the fact that $\widehat{R} \leq R^{o}$ and, under assumption $p_{H}^{2}>\left(1+\frac{1}{\delta q}\right) p_{L}^{2}, R^{o} \leq \frac{1}{p_{L}} \sqrt{2 \underline{U}}$ (by Claim 1 in the proof of Theorem 1).

Claim 3: $E \pi_{H}^{S T}\left(\widehat{R}, \widehat{w}_{J}^{S T}, \widehat{U}_{H}\right)=E \pi_{L}^{S T}\left(\widehat{R}, \widehat{w}_{J}^{S T}, \underline{U}\right) \geq E \pi^{L T}(\widehat{R})$.

Proof of Claim 3. The first equality corresponds to the definition of $\widehat{U}_{H}$. To prove the inequality, we notice that $\widehat{R} \leq \frac{1}{\widetilde{p}} \sqrt{2 \underline{\underline{U}}}$, because $\widehat{R} \leq R^{o}$ and $R^{o} \leq \frac{1}{\widetilde{p}} \sqrt{2 \underline{\underline{U}}}$ (by Claim 1 in the proof of Theorem 1). Given that $\widehat{R} \leq \frac{1}{\tilde{p}} \sqrt{2 \underline{\underline{U}}}$ and $\widehat{R} \leq \frac{1}{p_{L}} \sqrt{2 \underline{U}}$, the inequality can be written as $-\frac{1}{\delta} \widehat{w}_{J}^{S T}+\frac{1}{2}\left(p_{L} \widehat{R}\right)^{2} \geq-\frac{1}{\delta} \underline{U}+\frac{1}{2}(\widetilde{p} \widehat{R})^{2}$. By Claim 1, a sufficient condition is $-\frac{1}{\delta}\left(\underline{U}-\frac{1}{2} \delta q \widehat{R}^{2}\left(p_{H}^{2}-p_{L}^{2}\right)\right)+\frac{1}{2}\left(p_{L} \widehat{R}\right)^{2} \geq-\frac{1}{\delta} \underline{U}+\frac{1}{2}(\widetilde{p} \widehat{R})^{2}$, that is, $q\left(p_{H}^{2}-p_{L}^{2}\right)+p_{L}^{2} \geq \widetilde{p}^{2}$, which holds with equality.

Claim 4: $E \widetilde{\pi}_{L}^{S T}\left(R, \widehat{w}_{J}^{S T}, \underline{U}\right)>\max \left\{E \widetilde{\pi}_{H}^{S T}\left(R, \widehat{w}_{J}^{S T}, \widehat{U}_{H}\right), E \widetilde{\pi}^{L T}(R)\right\}$, for any $R<\widehat{R}$. Proof of Claim 4: It follows from Claim 3 and Lemma 1 (a) and (b).

Claim 5: $E \widetilde{\pi}_{H}^{S T}\left(R, \widehat{w}_{J}^{S T}, \widehat{U}_{H}\right) \geq \max \left\{E \widetilde{\pi}_{L}^{S T}\left(R, \widehat{w}_{J}^{S T}\right), E \widetilde{\pi}^{L T}(R)\right\}$, for any $R \geq \widehat{R}$. Proof of Claim 5: $E \widetilde{\pi}_{H}^{S T}\left(R, \widehat{w}_{J}^{S T}, \widehat{U}_{H}\right) \geq E \widetilde{\pi}_{L}^{S T}\left(R, \widehat{w}_{J}^{S T}\right)$ for any $R \geq \widehat{R}$ because of Claim 3 and Lemma 1 (b). The inequality $E \widetilde{\pi}_{H}^{S T}\left(R, w_{J}^{o}, \widehat{U}_{H}\right) \geq E \widetilde{\pi}^{L T}(R)$ for any $R \geq \widehat{R}$ also follows from Claim 3 and Lemma 1 (c) provided either $\widehat{U}_{H}>\frac{\widetilde{p}^{2}}{p_{H}^{2}} \underline{\underline{U}}$ or $p_{H}^{2}>2 \widetilde{p}^{2}$. We notice that $\widehat{U}_{H}$ is certainly higher than $\frac{\widehat{p}^{2}}{p_{H}^{2}} \underline{U}$ if $\widehat{R}$ is lower than but closer to $R^{o}$. Indeed, if $\widehat{R}=R^{o}$ then, following the same steps as in Claim 2 in the proof of Theorem 1 we obtain $\widehat{U}_{H}=U_{H}^{o}=\left(1+\frac{1}{\delta q}\right) \underline{U}$, higher than $\frac{\widetilde{p}^{2}}{p_{H}^{2}} \underline{\underline{U}}$ if and only if $\delta q p_{H}^{2}>(1+\delta q) \widetilde{p}^{2}$, which always holds.

Proof of Theorem 3. We recall that $R^{o}$ is characterised by $E \tilde{\pi}^{L T}\left(R^{o}\right)=E \tilde{\pi}_{L}^{S T}\left(R^{o}, 0, \underline{U}\right)$. If $R^{o}<\underline{R}$, then $R^{o}<R$ for all $R \in[\underline{R}, \bar{R}]$. Therefore, Lemma 1 (b) implies $E \widetilde{\pi}^{L T}(R)>$ $E \widetilde{\pi}_{L}^{S T}(R, 0, \underline{U})$ for all $R \in[\underline{R}, \bar{R}]$. It easily follows that $E \widetilde{\pi}^{L T}(R)>E \widetilde{\pi}_{L}^{S T}\left(R, w_{J}, U_{L}\right)$ for all $R \in[\underline{R}, \bar{R}], w_{J} \geq 0$ and $U_{L} \geq \underline{U}$. Therefore, in equilibrium, no ST contract can be signed, since it would imply that some firms choose the strategy of keeping low-ability 
senior workers, which is dominated by the strategy of always offering LT contracts.

\section{References}

[1] Anderson, A., Smith, L., 2010. Dynamic Matching and Evolving Reputations. Rev. Econ. Stud. 77 (1), 3-29. doi: 10.1111/j.1467-937X.2009.00567.x

[2] Alonso-Paulí, E., Pérez-Castrillo, D., 2012. Codes of Best Practice in Competitive Markets for Managers. Econ. Theory 49 (1), 113-141. doi: 10.1007/s00199-010-0537-y

[3] Becker, G.S., 1973. A Theory of Marriage: Part I. J. Polit. Econ. 81, 813-846.

[4] Chao, H., Wilson, E.R., 1987. Priority Service: Pricing, Investment, and Market Organization. Amer. Econ. Rev. 77, 899-916.

[5] Chiappori, P.A., Macho, I., Rey, P., Salanie, B., 1994. Repeated Moral Hazard: The Role of Memory, Commitment, and Access to Credit Markets. Europ. Econ. Rev. 38, $1527-1553$.

[6] Dam, K., Pérez-Castrillo, D., 2006. The Principal Agent Market. Front. Econ. Theory, Berkeley Electronics 1. (Republished in BePress Advances in Theoretical Econ.).

[7] Eeckhout, J., Weng, X., 2010. Assortative Learning. W. P. U. Pennsylvania.

[8] Ghatak, M., Morelli, M. and Sjöström, T., 2001. Occupational Choice and Dynamic Incentives. Rev. Econ. Stud. 68, 781-810. doi: 10.1111/1467-937X.00190

[9] Ghosh, S., Waldman, M., 2010. Standard Promotion Practices versus Up-or-out Contracts. Rand J. Econ. 41, 301-325. doi: 10.1111/j.1756-2171.2010.00101.x

[10] Hoppe, H.C., Moldovanu, B., Ozdenoren, E., 2011. Coarse Matching with Incomplete Information. Econ. Theory 47, 75-104. doi: 10.1007/s00199-009-0514-5

[11] Katz, L.F., Autor, D.H., 1999. Changes in the Wage Structure and Earnings Inequality. Handb. Labor Econ. 3: Part A, 1463-1555. doi: 10.1016/S1573-4463(99)03007-2

[12] Lambert, R., 1983. Long-Term Contracts and Moral Hazard. Bell J. Econ. 14, 441452. 
[13] Loh, E.S., 1994. Employment Probation as a Sorting Mechanism. Indus. and Lab. Rel. Rev. 47, 471-486.

[14] Malcomson J., Spinnewyn, F., 1988. The Multiperiod Principal-Agent Problem. Rev. Econ. Stud. 55, 391-408.

[15] Matutes, C., Regibeau P., Rockett K., 1994. Compensation Schemes and Labor Market Competition: Piece Rate versus Wage Rate. J. Econ. \& Manage. Strateg. 3, 325-353. doi: 10.1111/j.1430-9134.1994.00325.x

[16] Png, I.P.L., Samila, S., 2013. Trade Secrets Law and Engineer/Scientist Mobility: Evidence from "Inevitable Disclosure". W. P. Nat. U. Singapore.

[17] Rogerson, W., 1985. Repeated Moral Hazard. Econometrica 53, 69-76. doi:10.2307/1911724

[18] Roth, A., Sotomayor, M., 1990. Two-Sided Matching: A Study in Game-Theoretic Modeling and Analysis. Cambridge University Press, New York and Melbourne, Econometric Society Monographs.

[19] Serfes, K., 2008. Endogenous Matching in a Market with Heterogeneous Principals and Agents. Int. J. Game Theory 36, 587-619. doi 10.1007/s00182-007-0109-y

[20] Shapley, Ll.S., Shubik, M., 1972. The Assignment Game I: The Core. Int. J. Game Theory 1, 111-130.

[21] Terviö, M., 2009. Superstars and Mediocrities: Market Failure in the Discovery of Talent. Rev. Econ. Stud. 76, 829-850. doi: 10.1111/j.1467-937X.2008.00522.x 